\title{
A dynamic credit risk assessment model with data mining techniques: evidence from Iranian banks
}

\author{
Saba Moradi ${ }^{1}$ and Farimah Mokhatab Rafie ${ }^{2^{*}}$
}

\author{
* Correspondence: \\ F.mokhatab@modares.ac.ir \\ ${ }^{2}$ Tarbiat Modares University, Tehran, \\ Iran \\ Full list of author information is \\ available at the end of the article
}

\begin{abstract}
Giving loans and issuing credit cards are two of the main concerns of banks in that they include the risks of non-payment. According to the Basel 2 guidelines, banks need to develop their own credit risk assessment systems. Some banks have such systems; nevertheless they have lost a large amount of money simply because the models they used failed to accurately predict customers' defaults. Traditionally, banks have used static models with demographic or static factors to model credit risk patterns. However, economic factors are not independent of political fluctuations, and as the political environment changes, the economic environment evolves with it.

This has been especially evident in Iran after the 2008-2016 USA sanctions, as many previously reliable customers became unable to repay their debt (i.e., became bad customers). Nevertheless, a dynamic model that can accommodate fluctuating politicoeconomic factors has never been developed. In this paper, we propose a model that can accommodate factors associated with politico-economic crises. Human judgement is removed from the customer evaluation process. We used a fuzzy inference system to create a rule base using a set of uncertainty predictors. First, we train an adaptive network-based fuzzy inference system (ANFIS) using monthly data from a customer profile dataset. Then, using the newly defined factors and their underlying rules, a second round of assessment begins in a fuzzy inference system.

Thus, we present a model that is both more flexible to politico-economic factors and can yield results that are max compatible with real-life situations. Comparison between the prediction made by proposed model and a real non-performing loan indicates little difference between them. Credit risk specialists also approve the results. The major innovation of this research is producing a table of bad customers on a monthly basis and creating a dynamic model based on the table. The latest created model is used for assessing customers henceforth, so the whole process of customer assessment need not be repeated. We assert that this model is a good substitute for the static models currently in use as it can outperform traditional models, especially in the face of economic crisis.
\end{abstract}

Keywords: Fuzzy clustering, Non-performing loan, Credit risk, FIS, Dynamism, ANFIS

\section{Introduction}

A commercial bank, hereafter referred to as a bank, is a type of financial institution that provides services such as accepting deposits, making business loans, and offering basic investment products. Inevitably, banks must take risks in giving loans and credit

(c) The Author(s). 2019 Open Access This article is distributed under the terms of the Creative Commons Attribution 4.0 International License (http://creativecommons.org/licenses/by/4.0/), which permits unrestricted use, distribution, and reproduction in any medium, provided you give appropriate credit to the original author(s) and the source, provide a link to the Creative Commons license, and indicate if changes were made. 
cards to customers because these are economic drivers. This is delicate because a bank's survival is tied to taking appropriate risks; a non-risk-taking bank is as vulnerable as an overly-risk-taking one (Narindra Mandalaa \& Fransiscus, 2012). Therefore, when banks are faced with a risk, appropriate risk management depends on identifying, understanding, measuring, and finally providing appropriate strategies towards it (Bekhet \& Eletter, 2014). According to the Basel 2 accord, credit risk is one of the risks that banks face in allocating resources. It is defined as the probability of non-payment or delayed payment by customers or their inability to repay a loan (Cisko \& Klieštik, 2013). Customers with a high probability of loan repayment are classified in the good customer group and customers with a high probability of default are classified in the bad customer group (Akkoc, 2012).

Credit risk assessment is vital for banks; they must ensure that borrowers are able to pay their installments before allocating a loan to them (Narindra Mandalaa \& Fransiscus, 2012). According to Basel 2, each bank needs to organize and develop its own internal credit scoring system with which they can analyze a borrower's risk. This has led to an upsurge in the demand for scoring systems that can accurately model risks at high resolution; some institutions are remunerated very well to develop such models for banks upon request. These credit-scoring techniques can then be used as decision support tools or as automated decision algorithms for a wide range of customers (Heiat, 2012). Most current credit risk models have been developed based on trial and error and lack a theoretical framework (Wang et al., 2014). Moreover, most of these models are static and are unable to function efficiently in economic crises. Traditionally, banks have used static modeling frameworks to assess customer credit risks; however, the lack of responsiveness to the evolving economic environment renders these models inefficient, especially in the face of concept drifts, where a portion of previously good costumers fall into default (i.e., become bad customers). While, the traditional static models have proven to work reasonably well during periods of stasis, they fail to do so in the face of economic and political fluctuations. This is especially evident in Iran, after the governing regime (the Islamic Republic) came under several international political and economic sanctions. Consequently, the number of non-performing loans (NPLs) increased and many Iranian customers became unable to repay their obligations. As new factors were introduced during this period, the model criteria needed to be updated, as well. Opting for appropriate factors that work well in all circumstances is difficult (if not impossible); therefore, a dynamic model that can accommodate new factors is desirable. In this paper, we introduce a new model that can accommodate changing uncertain factors as well as the more stable certain factors used in static models.

\section{Background}

Analyzing credit risk is a pattern recognition problem (Kruppa \& Schwarz, 2013) and includes functions for predicting whether or not a customer will pay off a loan (Emel et al., 2003); therefore, the most important features are resolution and accuracy. Credit scoring evaluation used to focus primarily on delinquencies. In recent years, however, loss given default (LGD) and exposure have been among its most important criteria.

In this field, researchers have tried to solve the customer credit risk assessment problem, each using a different approach and technique; and each of them has tried to 
present a more accurate model than the others (see Table 1). With the advances made in computer technology, data collection and manipulation has become more feasible than ever; consequently, the demand for data analysis and data classification has increased (Zanin et al., 2016). Machine learning and data mining are among the most popular techniques used in this area. The latter refers to mining data in order to recognize its hidden patterns and relationships (Sumathi \& Sivanandam, 2006). Systems such as artificial intelligence, which reveal patterns in a database, are called data mining systems (Saitta et al., 2008).

Table 1 Proposed models of credit risk. The researchers have tried to solve the customer credit risk assessment problem, using a different approach and technique, and each of them has tried to present a more accurate model than others. Table 1 summarizes these researches. It shows that 90.9\% of the previous models are static and only $9.1 \%$ of them are dynamic

\begin{tabular}{|c|c|c|c|}
\hline Authors' name & Techniques & Static / Dynamic & $\begin{array}{l}\text { Type of predictor } \\
\text { (Certain/Uncertain) }\end{array}$ \\
\hline Hussain Ali Bekhet et al., 2014 & ANN: RBF & Static & Certain \\
\hline $\begin{array}{l}\text { Gusti Ngurah Narndra Mandalaa. } \\
\text { Et al, } 2012\end{array}$ & Decision Tree: c 5.0 & Static & Certain \\
\hline Weimin Chen et al., 2012 & Clustering, SVM & Static & Certain \\
\hline $\begin{array}{l}\text { Fatemeh Nemati Koutanaei } \\
\text { et al., } 2015\end{array}$ & GA, SVM, PCA & Static & Certain \\
\hline Zhiwang Zhang et al., 2014 & $\begin{array}{l}\text { Kernel, fuzzification, penalty } \\
\text { factors }\end{array}$ & Static & Certain \\
\hline Danenas P et al., 2015 & SVM & Static & Certain \\
\hline Ghodselahi, 2011 & SVM, Fuzzy clustering & Static & Certain \\
\hline Shahrzad Zaman, 2013 & MLP, RBF & Static & Certain \\
\hline Vahid Baradaran, 2015 & ANFIS & Static & Certain \\
\hline Blanco et al., 2013 & $\begin{array}{l}\text { MLP, linear discriminant analysis, } \\
\text { quadratic discriminant analysis, } \\
\text { logistic regression }\end{array}$ & Dynamic & Certain \\
\hline Bensic et al., 2005 & $\begin{array}{l}\text { Logistic regression, NNs and } \\
\text { Classification and regression trees }\end{array}$ & Static & Certain \\
\hline West, 2000 & $\begin{array}{l}\text { Linear discriminant, analysis and } \\
\text { logistic regression, decision trees, } \\
\text { kernel density estimation, nearest } \\
\text { neighbor }\end{array}$ & Static & Certain \\
\hline Witkowska et al., 2004 & $M L P, R B F$ & Static & Certain \\
\hline Yeh et al., 2009 & $\begin{array}{l}\text { K-nearest neighbor (KNN) classifiers, } \\
\text { Logistic regression, Discriminant } \\
\text { analysis, Nal̈ ve Bayesian classifier, } \\
\text { Classification trees, ANN }\end{array}$ & Static & Certain \\
\hline Ping et al., 2011 & SVM & Static & Certain \\
\hline Tsai, 2008 & MLP & Static & Certain \\
\hline Twala, 2010 & $\begin{array}{l}\text { ANN, decision tree, Naïve Bayes, } \\
\text { k-NN,logistic, discriminant analysis }\end{array}$ & Static & Certain \\
\hline Hsieh et al., 2010 & $\begin{array}{l}\text { NN, support vector machine, and } \\
\text { Bayesian network }\end{array}$ & Static & Certain \\
\hline Paleologo et al., 2010 & $\begin{array}{l}\text { SVM, decision trees, KNN, AdaBoost, } \\
\text { Subagged classifiers }\end{array}$ & Static & Certain \\
\hline Wang et al., 2011 & SVM & Static & Certain \\
\hline Oreski et al., 2014 & $\begin{array}{l}\text { Presented a new hybrid GA with } \\
\text { ANN }\end{array}$ & Static & Certain \\
\hline Maria Rocha Sousa et al., 2016 & Generalized Additive Model (GAM) & Dynamic & Certain \\
\hline
\end{tabular}


There are several techniques for data mining, each with different capabilities: e.g., decision trees and rule induction, neural networks, fuzzy modeling, support vector machines (SVMs), k-nearest neighbors (k-NN), Bayesian networks (BNs), instance-based algorithms, and learning classifier systems (Berthold \& Hand, 2003). All of these techniques can be classified into one of three categories: a) classical statistics, b) artificial intelligence, and c) machine learning (Girjia \& Sirvatsa, 2006). Attempts to acquire knowledge using machines date back to the 1950s (see (Rosenblatt, 1958) and the references therein). Today, machine learning is used in a wide range of fields including speech and image recognition, and its algorithms facilitate many routines such as fraud detection, web searches, text-based sentiment analysis, image segmentation, object recognition, and credit scoring. Materials science pioneered machine learning in the 1990s, applying artificial neural networks (ANNs) and other methods to predict corrosion behavior (Rao \& Mukherjee, 1996).

Statistical learning concerns learning from existing data and includes two types: supervised and unsupervised learning. The approach of clustering, i.e., partitioning a dataset into groups of similar members, has been established (Kaufman \& Rousseeuw, 2009). To find appropriate machine learning techniques such as ensemble methods, Nanni et al. (Nanni \& Lumini, 2009) used Australian, German, and Japanese financial datasets. They found that the "random subspace" of the Levenberg- Marquardt neural net was the best method of classification (Nanni \& Lumini, 2009).

The models presented so far can be organized into two categories. The first category applies already existing models such as ANN and SVM. The second category proposes a new hybrid model based on the existing models. Many models have been presented; however, banks still require a model that calculates customer credit risk and decreases the amount of NPLs. Here we review some such models.

\section{Single-method model}

Mandala et al. (Narindra Mandalaa \& Fransiscus, 2012), identified factors at a rural bank- Bank Perkreditan Rakyat- that are necessary for assessing credit applications. Additionally, a decision tree model was proposed on the basis of data mining methodology. Aiming to reduce the number of NPLs, current decision criteria for credit risk assessment are evaluated.

The credit risk assessment model was applied to the bank PT BPR X in Bali, which contains 1082 lenders (11.99\%) with NPLs identified as bad loan cases. This brought PT BPR X into the category of poorly performing banks.

Data mining is used in developing a decision tree model for credit assessment as it can indicate whether the class of the request of lenders is of performing loan or NPL risk. Using C 5.0 methodology, a new decision tree model was generated. The model suggests new criteria for analyzing loan applications. The evaluation results show that, by applying this model, PT BPR X can reduce the amount of NPLs to less than 5\% and the bank can be consequently classified as a well-performing bank (Narindra Mandalaa \& Fransiscus, 2012). Abdou et al. (Abdou \& Pointon, 2009) considered the current credit-scoring approach, which is based on personal judgment. It is shown that, compared to the currently used judgment techniques, statistical scoring techniques provide more efficient classification results (Abdou \& Pointon, 2009). Furthermore, neural net 
models provide better average correct classification rates, but the optimal choice of technique depends on the misclassification cost ratio. For a lower cost ratio, a probabilistic neural net is preferred while, for a higher ratio, multiple discriminant analysis (MDA) is the preferred choice (Abdou \& Pointon, 2009). Thus, there is a role for MDA as well as for neural nets.

There is some evidence of statistically significant differences between advanced scoring models and conventional models (Abdou \& Pointon, 2009). Zamani (Zamani, 2011) studied customers' behavior patterns under two models: a multilayer perceptron network (MLP) and a neural network composed of radial basis functions (RBFs). Comparison of the two models showed that the MLP is better than RBFs in predicting the credit risk of legal clients (Zamani, 2011).

Bensic (Bensic et al., 2005) studied some important features of credit scoring in small-business lending by comparing the accuracy of logistic regression, neural networks (NNs), and classification and regression tree (CART) decision trees. The results showed that the probabilistic NN model achieves the highest "hit rate" and the lowest type I error (Bensic et al., 2005). West (West, 2000) investigated the accuracy of five NN models of credit scoring; namely, multilayer perceptron, mixture-of experts, RBF, learning vector quantization, and fuzzy adaptive resonance. The results showed that the mixture-of-experts and RBF neural network models are more sensitive than the multilayer perceptron approach (West, 2000). Yeh et al. (Yeh \& Lien, 2009) explored data mining methods in an attempt to find the most accurate and predictive methods for finding the probability of defaults. They found that artificial neural networks provide the most accurate estimation of the probability of default among the six data mining techniques examined. Based on this, they established a model called the sorting smoothing method (Yeh \& Lien, 2009).

\section{Hybrid model}

A hybrid data mining model of feature selection and ensemble learning classification algorithms was developed by Koutanaei et al. (Nemati Koutanaei et al., 2015) based on three stages. The first stage concerns data gathering and pre-processing. In the second stage, four feature selection (FS) algorithms, including principal component analysis (PCA), genetic algorithms (GA), information gain ratio, and relief attribute evaluation functions are employed. Here, parameters of the FS methods are set from the classification accuracy of the SVM classification algorithm. After selecting the appropriate model for each selected feature, they are applied to the base and ensemble classification algorithms. Accordingly, the best FS algorithm (along with its parameters) is indicated for the modeling stage of the proposed model. In the third stage, classification algorithms are employed for the prepared dataset of each FS algorithm. The results of the second stage revealed that the PCA algorithm is the best FS algorithm. In the third stage, the classification results showed higher accuracy achieved by the ANN adaptive boosting (AdaBoost) method (Nemati Koutanaei et al., 2015).

A novel multi-criteria optimization classifier based on kernel, fuzzy, and penalty factors was proposed by Zhang et al. (Zhang, 2014): a kernel function is used first to map the input points into a high-dimensional feature space; an appropriate fuzzy membership function is then introduced to a multi-criteria optimization classifier that 
associates it with each data point in the feature space; and finally, unequal penalty factors are added to the input points of the imbalanced classes. Thus, the effects of the aforementioned problems are reduced (Zhang, 2014). Rather than expending effort on determining which of the two models provides greater predictive capacity, Baixauli et al. (Baixauli et al., 2012) highlighted the importance of combining a structural model with an accounting model. In fact, recent literature indicates that there is no superiority of one approach over the other because they both capture different aspects of the risk of bankruptcy in companies, and they should be combined to improve credit risk management (Baixauli et al., 2012). Shahari et al. (Shahari et al., 2015) collected the annual panel data from 2005 to 2012 from 40 Islamic banks from 12 countries. They provided some policy recommendations that can result in further reduction of credit risks and improvement of bankers' confidence level in implementing asset-based financing policies (Shahari et al., 2015). Using a hybrid data mining technique, Chen et al. (Chen et al., 2012) proposed a credit-scoring model that has two processing stages: clustering and classification. In the clustering stage, samples of accepted and new applicants are divided into homogeneous clusters, the isolated samples are excluded, and inconsistent samples are relabeled. In the classification stage, samples with new labels are fed into SVMs to build the scoring model. One difference between this model and the other credit-scoring models is that the samples are classified into three or four classes rather than just "good" and "bad" classes. Based on the credit data set provided by a local bank in China, their experimental results showed that choosing a proper cut-off point can result in superior classification accuracy of good and bad customers. According to the characteristics of each class, risk management strategies are then developed (Chen et al., 2012). Research shows that defaults relate largely to macroeconomic variables (Yurdakul, 2014) and that uncertainty of economic policies increases banks' credit risks (Chi \& Li, 2017) with negative effects on loan size (Chi \& Li, 2017). Danenas (Danenas \& Garsva, 2015) proposed a credit risk evaluation method consisting of SVM classifier selection, external evaluation, and a sliding window. Results showed that the proposed method is comparable to other classifications like RBF networks and logistic regression (Danenas \& Garsva, 2015).

Ping et al. (Ping \& Yongheng, 2011) proposed a hybrid SVM-based model for evaluating credit scores based on customer variables which consists of four methods: (1) using a rough neighborhood to set input feature selections; (2) applying a grid search for optimizing RBF kernel parameters; (3) using hybrid optimal input features and a model; and (4) comparison between the accuracy of the suggested method and other methods. Results illustrated that the SVM-based hybrid classifier and the rough neighborhood set yield the best credit-scoring ability in comparison with other hybrid classifiers. They also outperformed linear discriminant analysis, logistic regression, and NNs (Ping \& Yongheng, 2011).

Combining classifiers is one of the concerns of recent research in machine learning. Twala (Twala, 2010) explored the predictions of five classifiers in credit risk predictions based on their manner of confronting noise and accuracy in applying classifier ensembles. He showed that the ensemble of classifiers can improve the accuracy of prediction (Twala, 2010). Hsieh et al. (Hsieh \& Hung, 2010) introduced a preprocessing step for obtaining an efficient ensemble classifier. They proposed class-wise classification using several data-mining techniques such as NN, SVM, and Bayesian networks to further increase the efficiency of the ensemble of classifiers (Hsieh \& Hung, 2010). 
Table 1 summarizes data-mining techniques and types of predictors.

It shows that $90.9 \%$ of the previous models are static and only $9.1 \%$ of them are dynamic. International sanctions were inflicted on the Iranian regime during 2008-2016. The number of NPLs increased (Fig. 1) and economic sectors were affected by the NPLs (Fig. 2). Our experiences with Iran showed that critical circumstances such as hyperinflation, sanctions, and unemployment can affect customers' lives and move them from the "good customer" segment to the "bad customer" segment.

Interestingly, more than 73\% of NPLs were backed loans (in which the borrower offers very large collaterals to secure the loan). Apparently, these collaterals could not guarantee customer repayment. The prevailing credit risk assessment models were inaccurate in critical situations like those of sanctions. Customer behavior is subject to change with the passage of time, and so is customer credit risk. A sophisticated dynamic model that can account for these kinds of crises is required.

A dynamical modelling framework for credit risk assessment was recently proposed by Sousa et al. (Sousa \& Gama, 2016); it extends the prevailing models developed on the basis of historical data static settings. This model was inspired by the principle of films, using "a sequence of snapshots, rather than a single photograph." In this dynamic modeling framework, customer credit risk is assessed using a batch data processing model. A generalized additive model (GAM) is used for classification of data in a supervised training environment and the learning units are set as static units. The researchers used a Gini coefficient for measuring model performance from data from previous months. The model deals with defaults in two ways: a full-memory time window based on all previous data, in which new data is appended to the training set (which is unable to adapt to major changes); and a fixed short-memory time window that forgets the past. The latter was used because the researchers believed that there is a low correlation between ongoing defaults and past instances. Figures 3 and 4 show the full- and short-memory time windows, respectively (Sousa \& Gama, 2016). Although this model has been proven to outperform static models in helping the banks to prevent probable future losses, it has some shortcomings. In the banking industry, credit-scoring models are usually developed from static windows and are kept unchanged for long periods of time, possibly years. Although, Sousa et al. (Sousa \& Gama, 2016) provided convincing results in their research, they did not consider some important topics in credit risk assessment. For example, their model considered a set of fixed

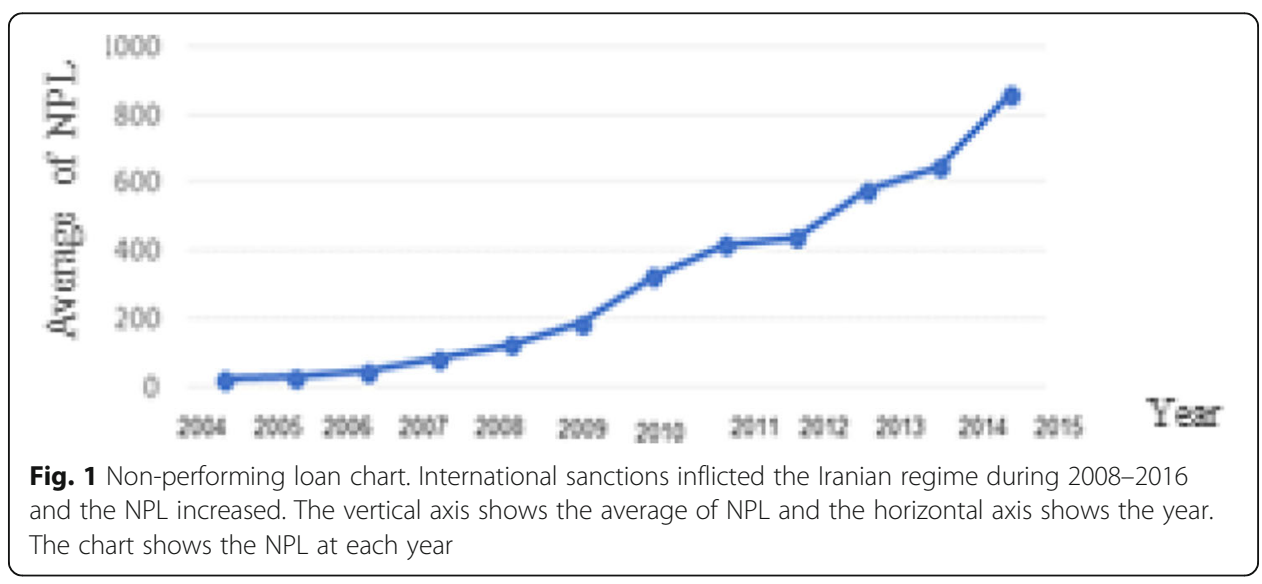




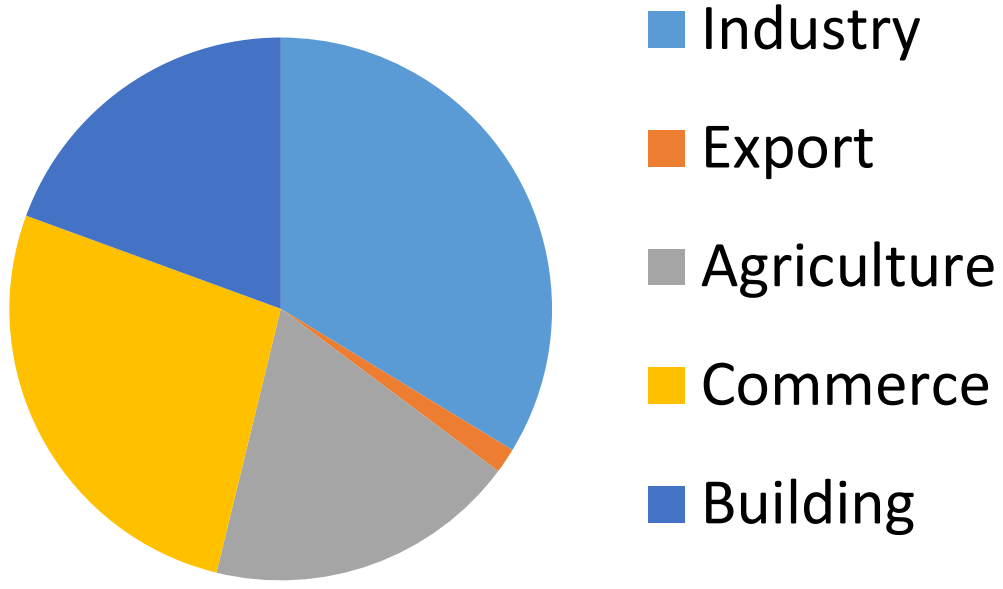

Fig. 2 The economic sector affected by the sanction. International sanctions have affected the economic sector such as industry, export, agriculture, commerce, and building

predictors even though they suggested that a "predictor of variable length" would be more effective in finding the reason behind NPLs (Figs. 3, 4 and 5).

In this research we propose a model that considers a wider array of factors than in the previous studies: a set of certain factors like age, marital status, monthly income, etc. and a set of uncertain predictors (Table 2). A group of ten top risk managers and banking specialists (credit risk workgroup) helped us to determine new factors based on their experiences and according to Basel 2. In this model, we construct a table of bad customers (i.e., those that have failed to repay their debts for over 2 months) on a

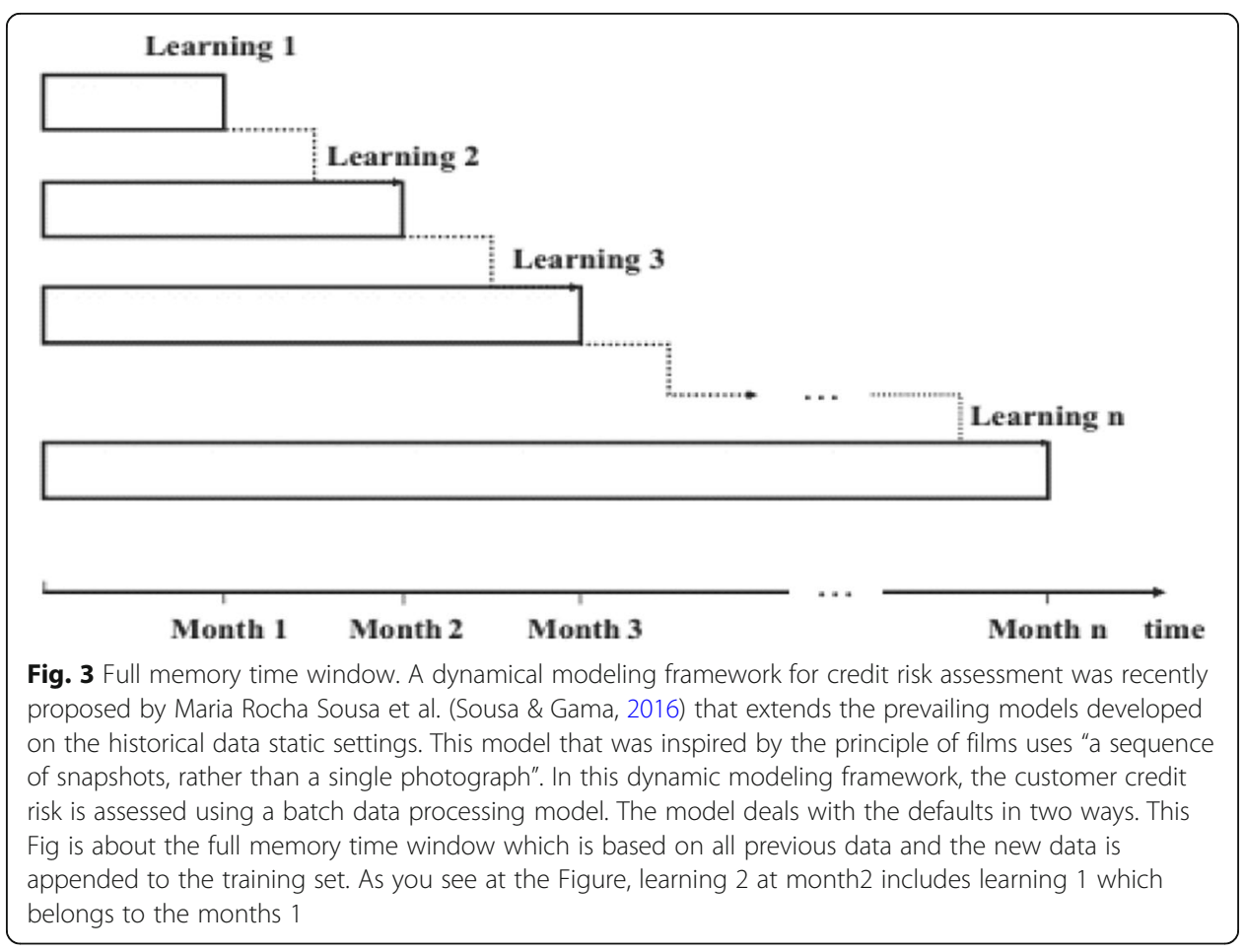




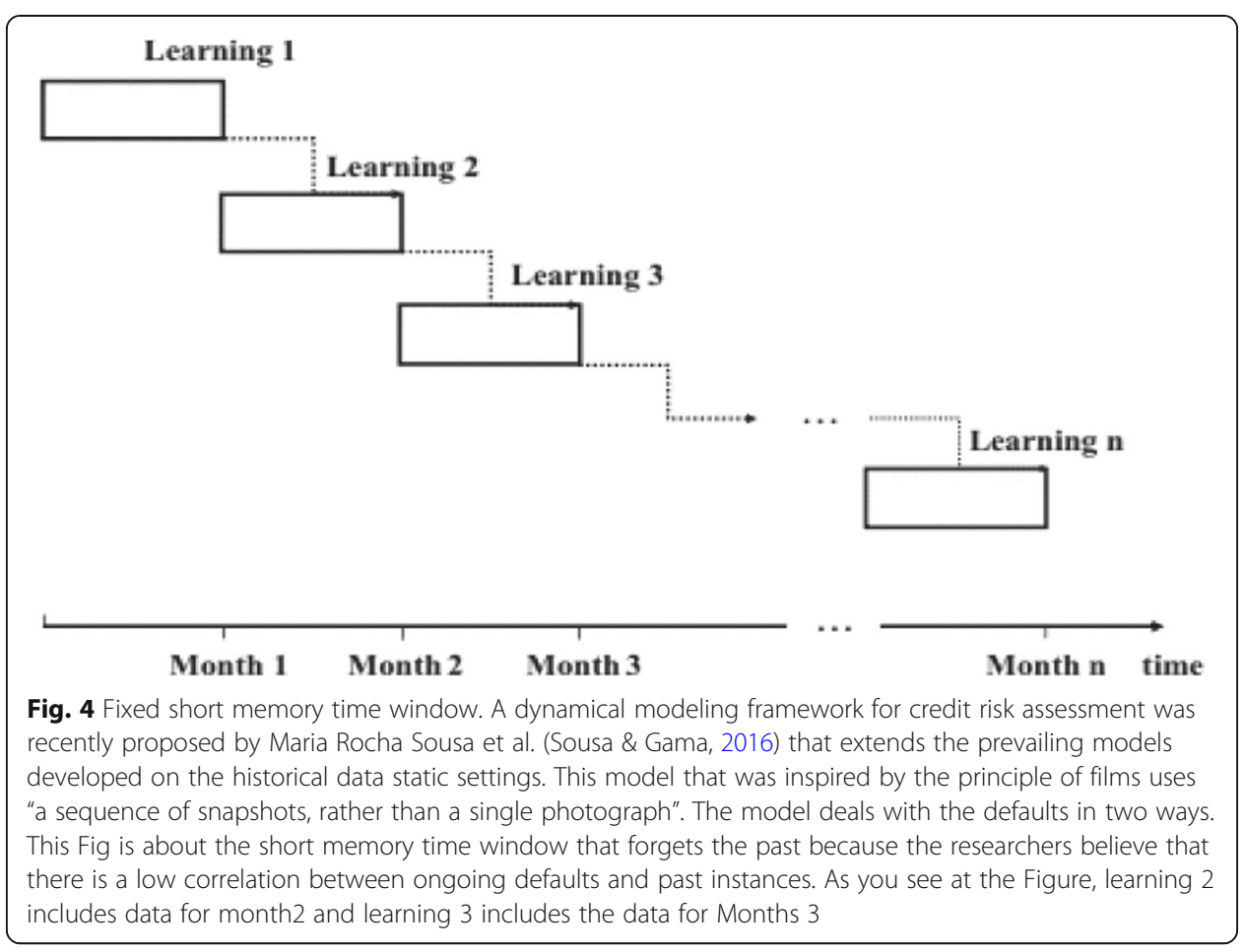

monthly basis; then we train the ANFIS with this data. A fuzzy inference system (FIS) then applies our defined rules to model the customers' defaults.

The conceptual diagram of the proposed model is shown in Fig. 6.

In lending, it is vital to rely on models instead of human judgement (Khandani \& Kim, 2010). In this model, human judgement is removed from the customer evaluation process. The factors have been chosen in such a way that they cluster customers better

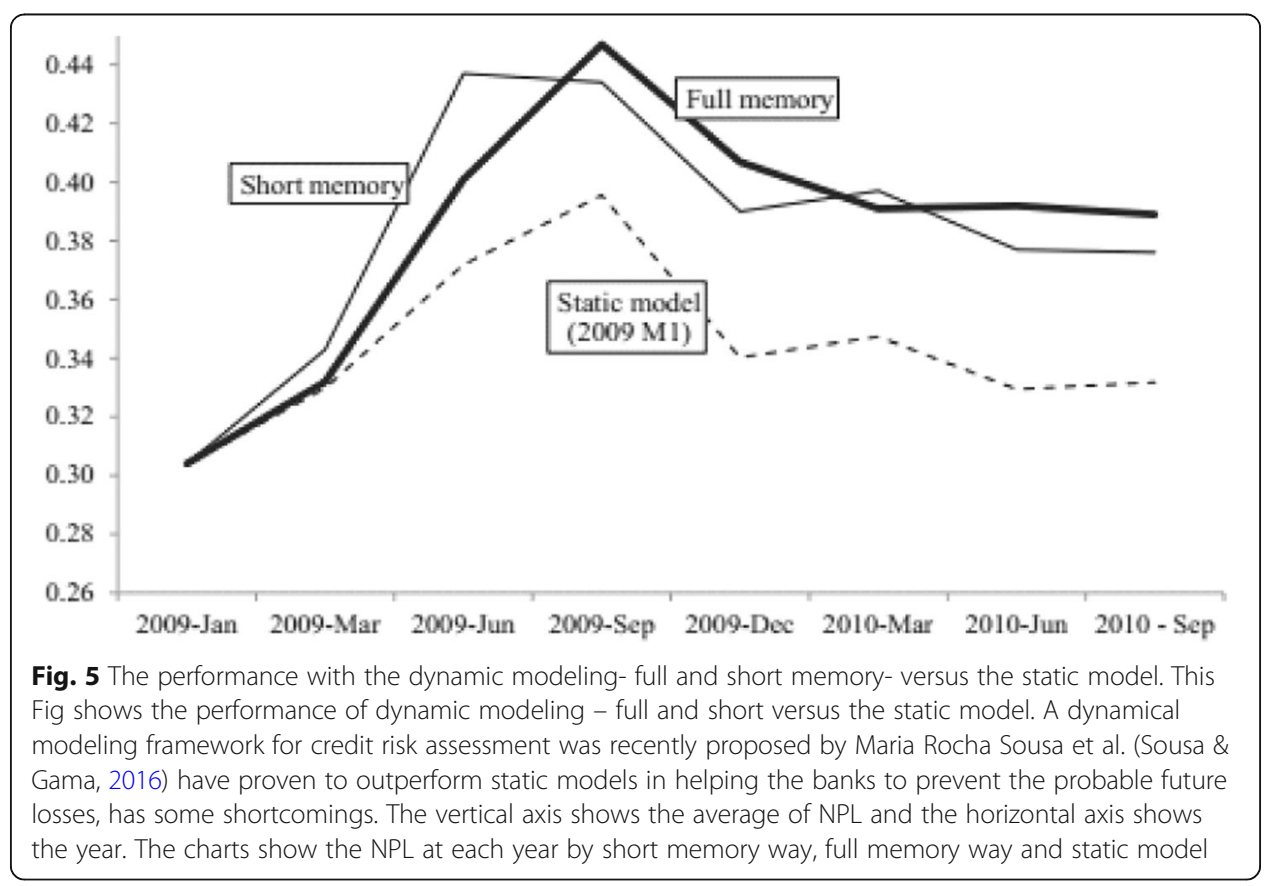


Table 2 Factors of the proposed model. In this research, we propose a model which considers a wider array of factors than the previous researches: a set of certain factors like age, marital status, monthly income, etc. And a set of uncertain predictors (Table 2). A group of 10 top risk managers and banking specialists (credit risk workgroup) helped us to determine these new factors base on their experiences and according to Basel 2

\begin{tabular}{|c|c|c|}
\hline Variables & $\begin{array}{l}\text { Type of customer that } \\
\text { corresponds to the variable }\end{array}$ & $\begin{array}{l}\text { Certain or } \\
\text { uncertain }\end{array}$ \\
\hline Number of customer product & Individual/legal customer & Certain \\
\hline Number of adverse judgment & Individual/legal customer & Certain \\
\hline Number of loan repayment past due & Individual/legal customer & Certain \\
\hline Non-performing amount & Individual/legal customer & Certain \\
\hline Number of hard inquiries & Individual/legal customer & Certain \\
\hline Number of rejected request & Individual/legal customer & Certain \\
\hline Number of dishonored cheque & Individual/legal customer & Certain \\
\hline Collateral valuation & Individual & Uncertain \\
\hline Number of risky customers guaranteed & individual & Certain \\
\hline Number of write off & Individual & Certain \\
\hline Duration of non-performing & Individual/legal customer & Certain \\
\hline Exposure amount for each customer & Individual/legal customer & Uncertain \\
\hline Expected loss & Individual/legal customer & Uncertain \\
\hline Deposit amount & Individual & Certain \\
\hline Debt to income ratio & Individual & Certain \\
\hline Credit score of guarantor & Individual/legal customer & uncertain \\
\hline Customer probability of default & Individual/legal customer & uncertain \\
\hline Capital return & legal customer & uncertain \\
\hline Arrears amount & legal customer & Certain \\
\hline Liability & Individual/legal customer & Certain \\
\hline Net flow & legal customer & Certain \\
\hline Average asset balance previous 12 month & legal customer & Certain \\
\hline Available credit amount & Individual/legal customer & Certain \\
\hline Number of repossessions & Individual/legal customer & Certain \\
\hline Pay hike/rise & legal customer & Certain \\
\hline Earning power & legal customer & Certain \\
\hline Earning asset & legal customer & Certain \\
\hline Intangible asset & legal customer & Certain \\
\hline Deferred income & legal customer & Certain \\
\hline Recovery rate & Individual/legal customer & Uncertain \\
\hline Due date & Individual/legal customer & Certain \\
\hline Post-dated check & Individual/legal customer & Certain \\
\hline Non mortgage credit payment & Individual & Certain \\
\hline Customer life time value & Individual/legal customer & Certain \\
\hline Occupation stability & Individual/legal customer & Uncertain \\
\hline
\end{tabular}

than the models currently in use. Dynamic clustering techniques were used for clustering. In the next section, we present a description of the main concepts such as fuzzy theory, the fuzzy inference system (FIS), and the adaptive network-based fuzzy inference system (ANFIS). Section 3 presents the research methodology as well as the 


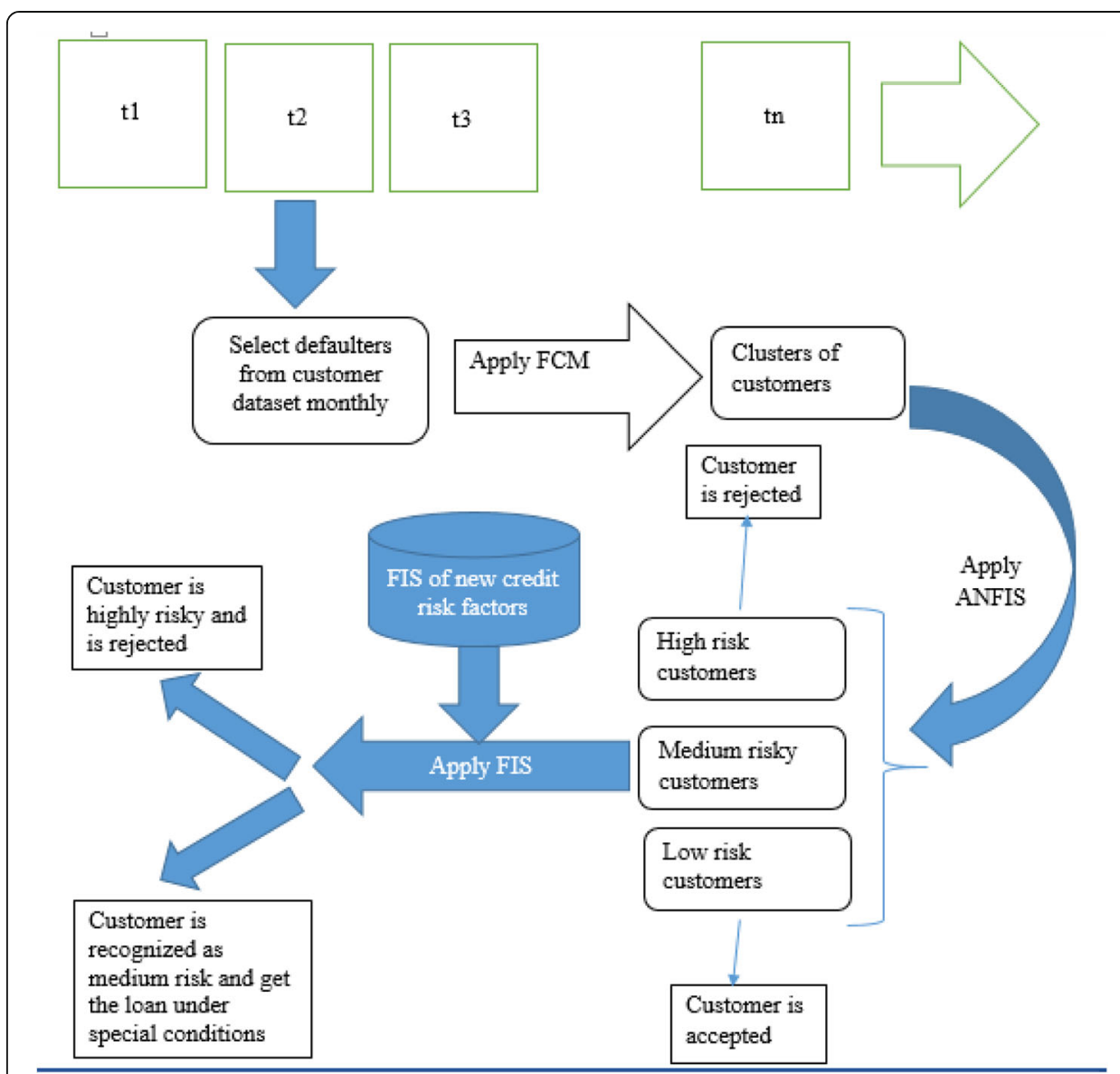

Fig. 6 Conceptual model of the dynamic model. $T 1, \mathrm{t} 2 \ldots$ th show the months. We organize a table based on the monthly data on bad customers (i.e. those who have not paid their debt for the last 2 months or so), then we apply FCM to cluster customers, then we train the ANFIS and construct a dynamic model based on the data in this table. This model is used then to assess the customers' credit risk at the time of registry. Only if the customer is assessed to be low risk, they are given credit. Otherwise, if they are found high risk they will get no credit. Alternatively, if the customer belonged to the medium-risk segment in the analysis with the dynamic model, the second round of assessment will begin using a fuzzy inference system based on our predefined rules. The analysis ends if the customer is still shown to be too risky. However, if they are shown to belong to the medium risk group, conditional credit can be allocated to these customers

dynamic model of this research. A case study and its solution is provided, as well. Section 4 includes the results and discussion of the study; finally, Section 5 concludes this paper.

\section{Fuzzy theory}

The word fuzzy in the Longman Dictionary of Contemporary English is defined to mean inaccurate and unclear (Procter, 1978). For over two thousand years, Aristotle's law has governed our perception of what is true and what is false, philosophically. In 1960, Professor Zade, a prominent scholar of control theory, presented fuzzy theory to explain real phenomena that are ambiguous and fuzzy. Unlike Boolean logic, which works based zeros and ones, fuzzy logic works based on the degree of membership of an element in a fuzzy set defined by a membership function. Figure 7 shows the 


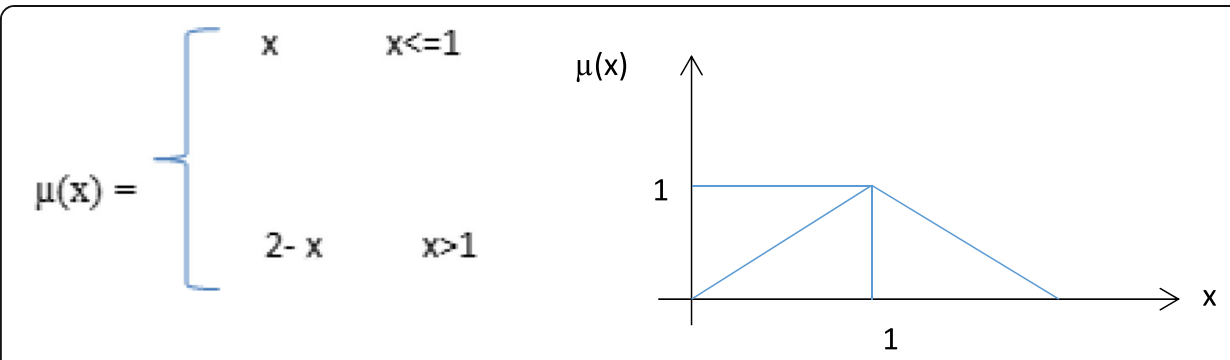

Fig. 7 Membership function of a real number. This Fig is the graphical representation of the membership function of the fuzzy set of real numbers near one. The horizontal axis is number and the vertical axis is the value of the membership. For example, the value of the membership of number one is one

graphical representation of the membership function of the fuzzy set of real numbers near one (Dikjkman et al., 1983).

To illustrate the difference between Aristotelian/Boolean and fuzzy logic (Fig. 8), consider the expression of people's height in fuzzy theory. The degree of height for individuals between 150 and $180 \mathrm{~cm}$ tall would appear as shown in Fig. 9.

Practical applications of fuzzy theory were initiated in the 1970s as skepticism about its existential nature was dispelled (see Amid (Amid, n.d.) and the references therein). Fuzzy theory has since become popular because it provides an appropriate tool for modeling complex and uncertain systems. Fuzzy logic has several suitable features that make it a flexible and powerful toolbox for dealing with inaccurate data (for a review of applications, see (Dikjkman et al., 1983)). Moreover, a fuzzy system can easily be established on the expertise of experienced people. Human opinions can be converted into rules using fuzzy theory. Therefore, since part of this research is based on expert knowledge, we used fuzzy logic (see the research methodology section).

\section{Fuzzy inference system (FIS)}

The fuzzy inference system (FIS) provides a systematic process for converting a knowledge-based system into a nonlinear mapping. The first component of the system is fuzzification, which converts the numerical values of input variables into a fuzzy set. The second component includes a fuzzy rule base that is a set of if-then rules and a fuzzy inference engine that converts the inputs into a series of outputs. Finally, a defuzzification mechanism that converts the fuzzy output into a definite number (Nauk et al., 1997) is applied. Figure 10 shows the steps of the fuzzy inference system.

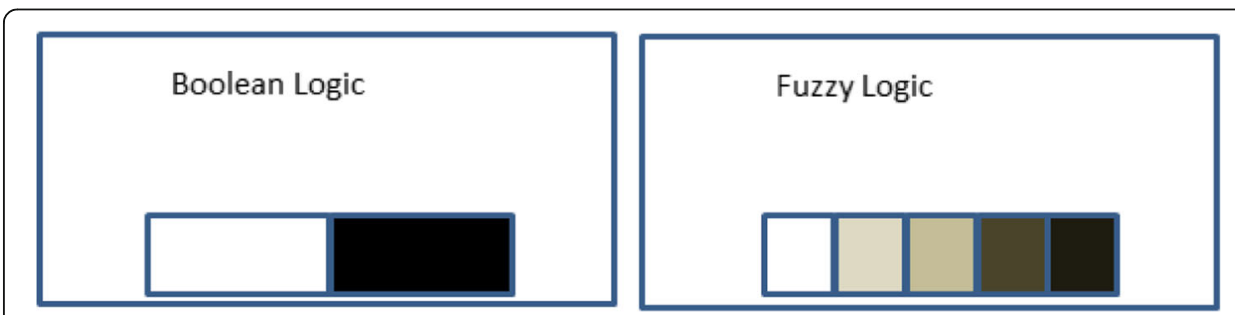

Fig. 8 Boolean logic and Fuzzy logic. This Fig illustrates the difference between Aristotelian/Boolean and fuzzy logic. Aristotelian logic has defined border between zero and one but Fuzzy logic does not have defined border. It is a range between zero and one 


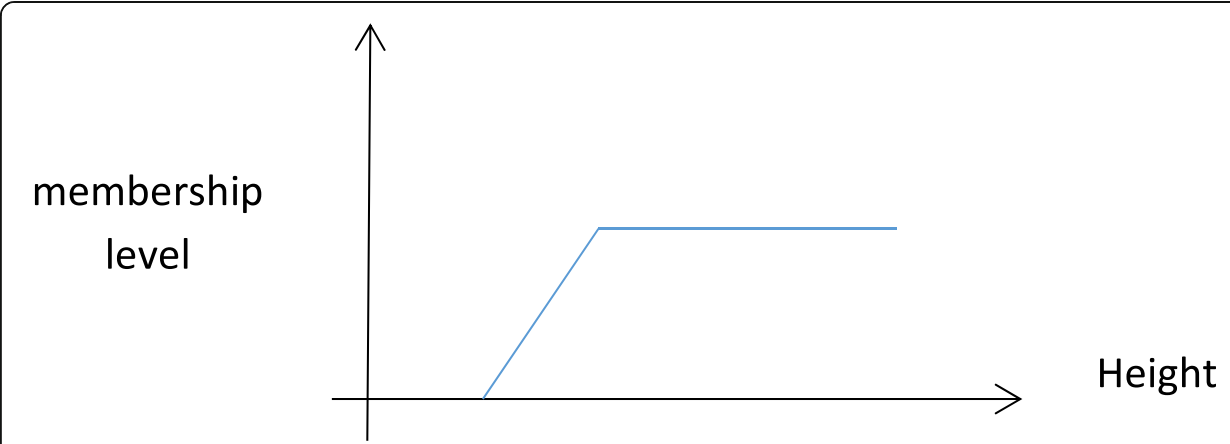

Fig. 9 Membership function of tallness. This Fig considers the expression of the people's height in the fuzzy theory. The degree of tallness for individuals between 150 and $180 \mathrm{~cm}$

Various methods have been used in the literature for fuzzifying and defuzzifying variables (Wang \& Chen, 2014). Through a series of trial and error, we chose the Sugeno method (Sugeno, 1985), in which the preceding expressions are fuzzy and yielded more accurate results.

\section{Adaptive network-based fuzzy inference system (ANFIS)}

Consider a system that looks like a black box. It receives some inputs and produces some outputs. The aim is to design a neuro-fuzzy model that accurately describes the system. According to Fig. 11, if the error is zero for every input, then the model works exactly like the system.

Jang (Jang, 1993), the inventor of this method, defined a function called mean squared error (MSE) and proved that, if the value of the cost function is minimized by changing the model parameters, the model approaches the real system. This process is called training. There is a theorem according to which, if there is a target system like that shown in Fig. 11, then a fuzzy system can be designed as closely as desired to the system. Different methods have been proposed for the implementation of Fig. 11, but the most important structure proposed so far is an adaptive network-based fuzzy

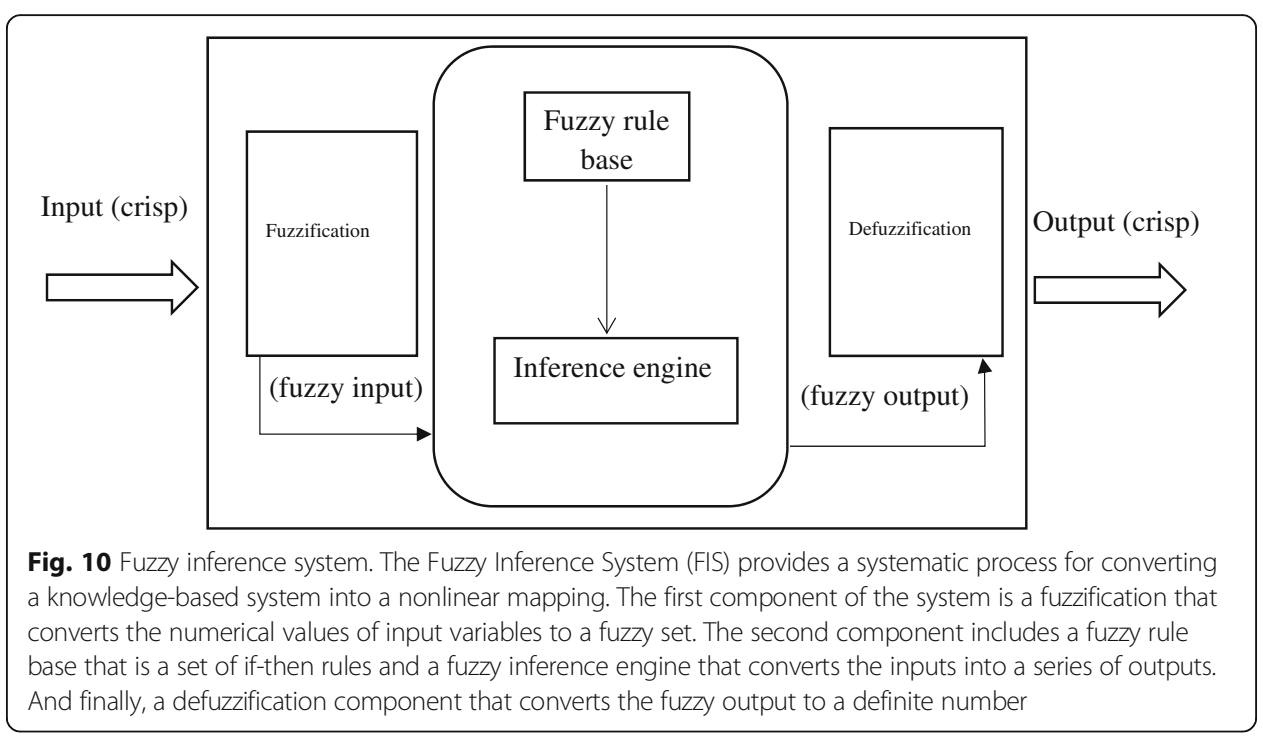




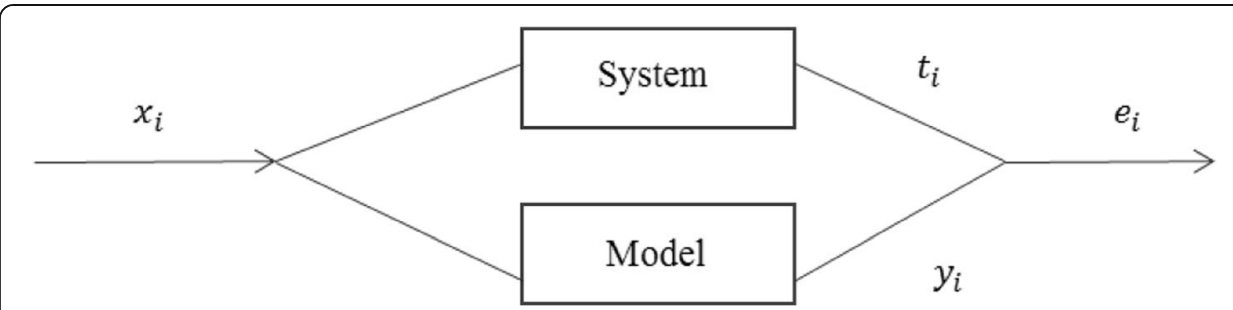

Fig. 11 System Figure. This Fig supposes a system like a black box. It gets $x_{i} s$ as inputs and gives $t_{i} s$ as outputs. The aim is to design a model of a neuro-fuzzy that describes the system accurately. If the error is zero for every input, then the model works exactly like the system

inference system (ANFIS). It adapts itself to the input data and gradually minimizes error based on the gradient descent training principle. An ANFIS is a comparative neural network offering the advantages of learning, optimization, and fuzzy logic.

An adaptive network is a network structure that connects several nodes to several links.

The nodes represent processing units and the links show the connection between those processing units. The rules of learning are made in a way to reduce system error and properly correct the node parameters. To determine the parameters, the ANFIS uses the hybrid learning principle, which combines the method of gradient descent and the least squares method.

Sugeno or Takagi-Sugeno-Kang is a method of fuzzy inference. Consider a Sugeno fuzzy model with two inputs and one output. The fuzzy rules can be set as follows:

If Input $1=x$ and Input $2=y$, then the Output is $z=a x+b y+c$ (formula 1 ).

The total output is the weighted average of outputs. The structure of the ANFIS is shown in Fig. 12 (Polat \& Günes, 2006).

\section{Research methodology}

\section{Approach}

Our model takes a straightforward route. We organize a table based on monthly data on bad customers (i.e., those who have not repaid their debts for the last 2 months or

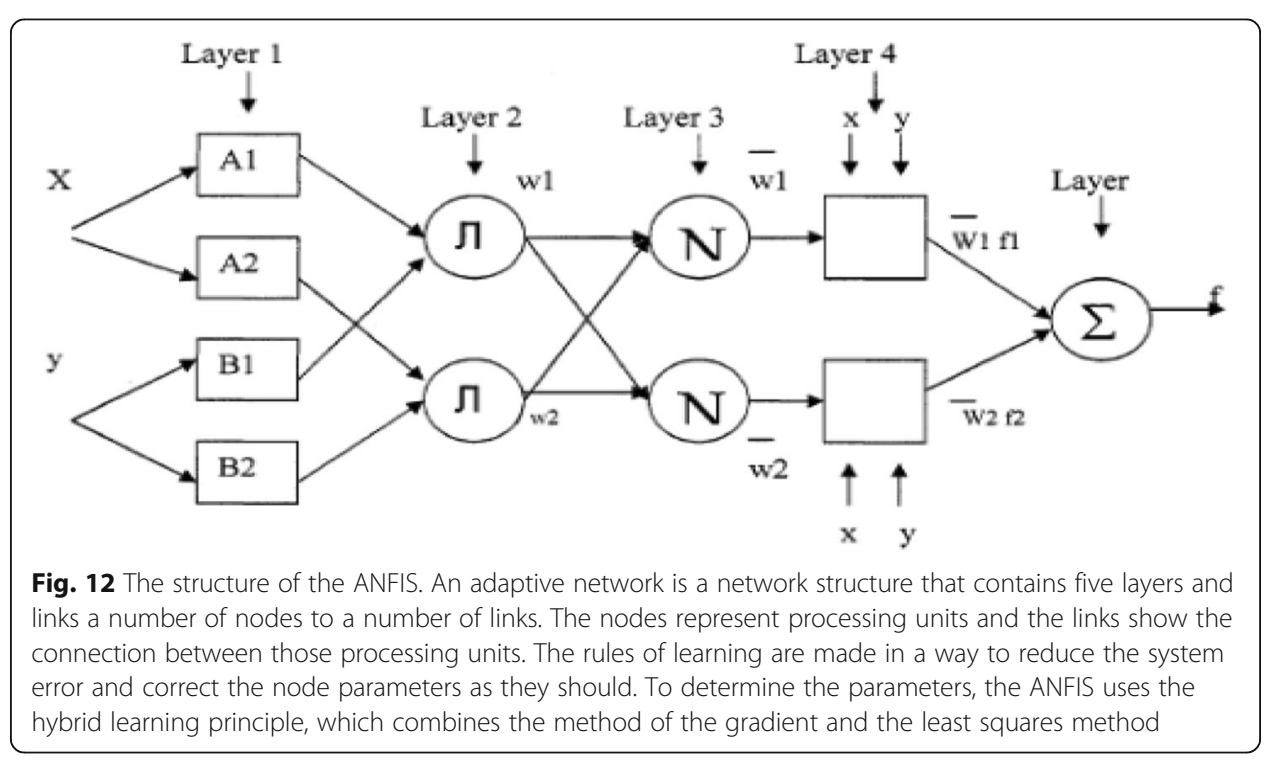


so); then we train the ANFIS and construct a dynamic model based on the data in this table. This model is then used to assess the customers' credit risk at the time of registry. Only if the customer is assessed to be risk free based on the static models from the dataset containing the information on all customers, is the customer given credit. Otherwise, if the customer is found to be too risky, the customer is given no credit. Alternatively, if the customer belongs to the medium-risk segment in the analysis using the dynamic model, a second round of assessment begins using a fuzzy inference system based on our predefined rules. The system classifies customers into three clusters of low, medium, and high risk. The analysis ends if the customer is still shown to be too risky. However, if the customer is shown to belong to the medium-risk group, conditional credit can be allocated; if classified in the low-risk group based on the second round of analysis, the customer is given credit and the analysis ends.

All calculations and the construction of FIS and ANFIS was done by the FIS and ANFIS toolboxes in MATLAB R2015b. However, for developing the model for a larger scale, Java and Oracle can be used. Figure 13 summarizes the research methodology.

\section{Dynamic model for credit risk}

Traditionally, researchers have applied methods like SVM, PCA, and ANN and focused mainly on repetitive demographic factors to forecast credit risk. While these models work reasonably well during periods of stasis, they cannot take economic crises into account. A model is needed that can account for the passage of time and critical situations like sanctions, which can have unfortunate impacts on customers' personal lives and, more importantly, on their ability to repay their obligations.

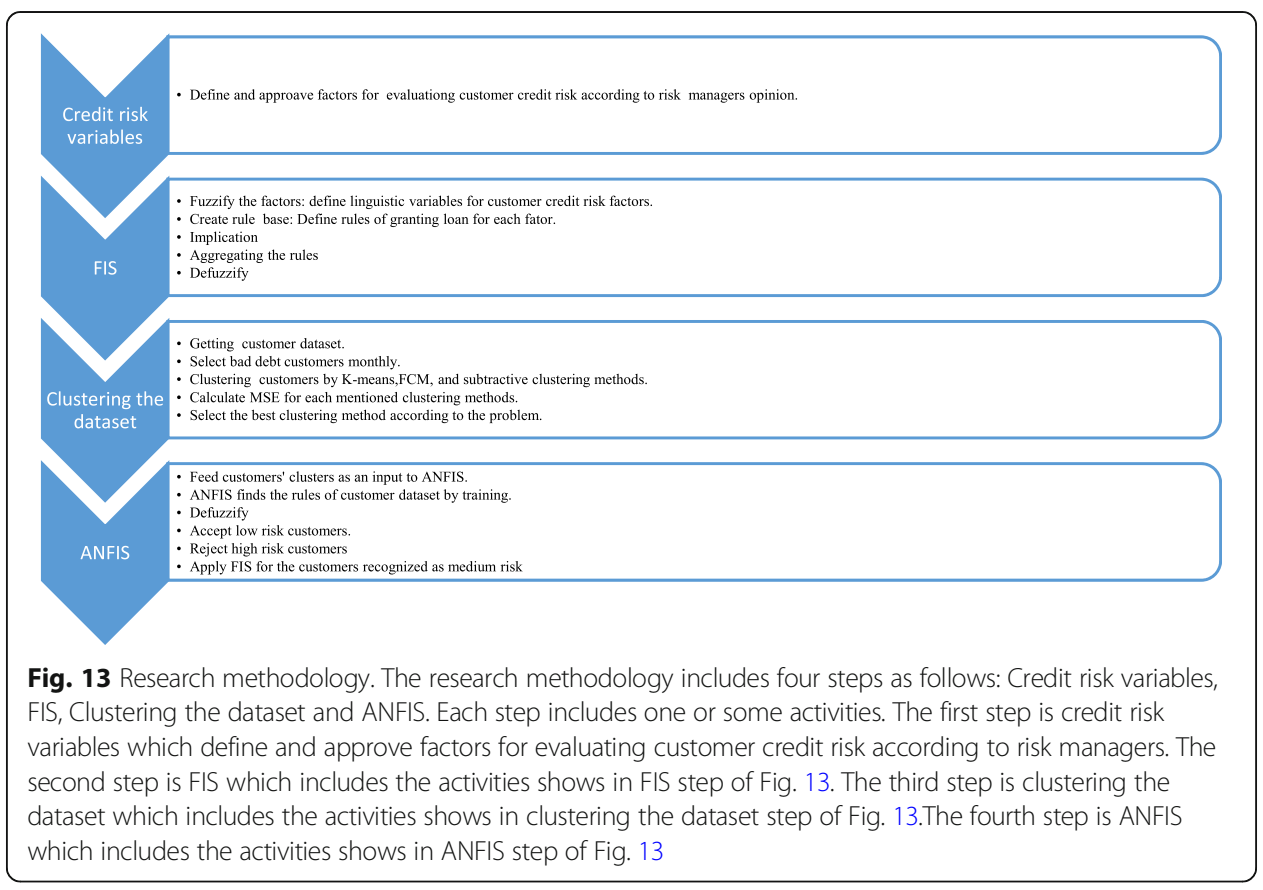


The default rate has grown at an alarming rate in Iran following the economic and political sanctions applied against the governing regime. This growth has been unpredictable in the static models that Iranian banks currently use. We combine FIS, fuzzy clustering, and ANFIS to create a dynamic model that is robust to these political and economic fluctuations. Therefore, the factors applied in this model are different from those of previous research, and can be used for both individuals and legal customers (see Table 2). We defined these factors and a group of ten top risk managers in several meetings, who approved them. Some of the factors do not change over time; we called these certain factors. Others do change; we called these uncertain factors. We applied fuzzy theory to the uncertain factors.

We used FIS and fuzzy theory to implement risk managers' opinions as a rule base for the dynamic model. The FIS contained the new credit risk factors and related rules between them.

Considering the behavioral features of customers in special economic and political conditions, fuzzy numbers and their related calculations can be applied in solving customer credit risk problems. Fuzzy systems have a unique capability in utilizing human knowledge and are appropriate tools for modelling complex systems dealing with uncertainty. As reviewed in the background section, ANNs cannot individually exploit human knowledge as they are a data driven method and need data; but fuzzy systems are knowledge-based systems. In ANNs, it is difficult to define a rule that can be used by a human. However, in a fuzzy system, it is possible to create a rule that is understandable and implementable by a human. We divide the customers into three groups based on how late they have been in paying instalments: low risk (LR), indicating less than 2 months; medium risk (MR), from 2 to 6 months; and high risk (HR), more than 6 months. According to the membership function concept, each customer belongs partly to each group and there are no definite boundaries between them.

\section{Dynamic engine of the proposed model}

The statistical population used in this study contains 9000 records of bank customer profiles in a database that includes properties like name, age, time at current address, monthly income, and application date, due date, instalment date, and number of products, gender, and names of the parents of the customers. Some behavioral patterns are clearly observable based on the database; however, these patterns have proved to change as the political and economic environment changes.

Moreover, training using all data from the customer dataset and constructing a dynamic model of credit risk that needs to be updated every few months is costly for banks and financial institutions; they usually decline to use such models. They prefer to construct a model once and use it for years. In order to determine the behavioral patterns of customers in a period of economic crisis, we collected data monthly on customers that failed to repay their debts for over 2 months and organized them into a table. Then we used this data in an ANFIS to create a new dynamic model. Jang (Sugeno, 1985) found that models developed via ANFIS, which is a general approximator, can be very close to reality. This model then became the dynamic engine of our model. 
The customer features with the most impact on the patterns were selected in this research; they include age, monthly income, number of dependents, marital status, occupation code, type of home, and bill payment experience.

As mentioned above, the input for ANFIS was the monthly customer profile dataset. Some underlying rules in the customer profile dataset are hidden from a human observer; therefore, we fuzzy-clustered the customers before feeding them into the ANFIS, thus letting the models recognize the rules better and decreasing the calculation load.

There are several available clustering methods like k-means, FCM, and subtractive approaches. To find which is best for our research, we clustered the customers using k-means, FCM, and subtractive clustering methods. The MSEs of three methods (k-means, FCM, and subtractive clustering) are shown respectively in Figs. 14, 15, and 16.

Figure 15 shows that the FCM yields the best results. The k-means method had a crisp border between the three clusters but most of the risks occurred along the borders. How best to behave with these borderline customers is an important problem.

The results of FCM analysis were the best because it changed to adopt milder behavior at the borders. The subtractive method clustered customers into several segments and did not fit the purpose of the model.

The customer dataset was clustered into three segments fed into the ANFIS as input. After training the ANFIS, the underlying hidden rules of the system became evident. We first clustered the data set into manageable segments using an unsupervised fuzzy clustering method because it assumed no definite boundaries between the customer segments. The unsupervised approach was taken because we wanted the system to cluster customers without any bias. This network can adapt itself over time and can discover the rules of the system.

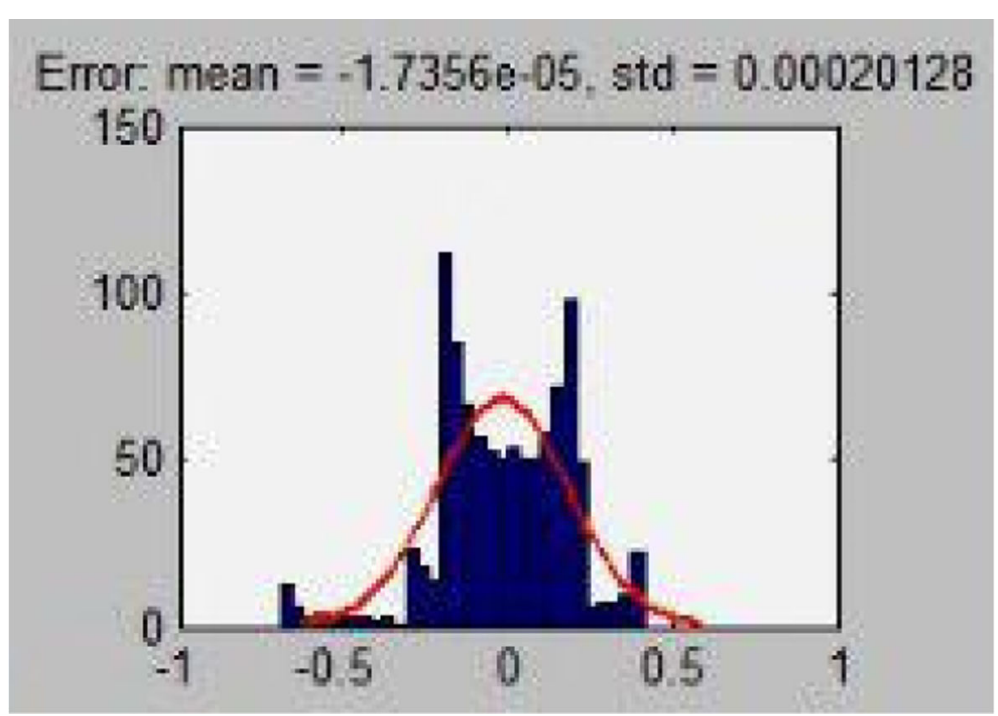

Fig. $14 \mathrm{~K}$-means MSE. There are some clustering methods like K-Means, FCM, and subtractive. To find which one is the best for our research, we have clustered the customers using K-Means, FCM, and subtractive clustering methods and compared the methods' MSEs. The MSEs (Mean-Square Error) of the k-means is shown in Fig. 14. The error mean is $=-1.7356 \mathrm{e}-0.5$ and $s t d .=0.00020128$. The Mean squared error (MSE) measures the average of the squares of the errors 


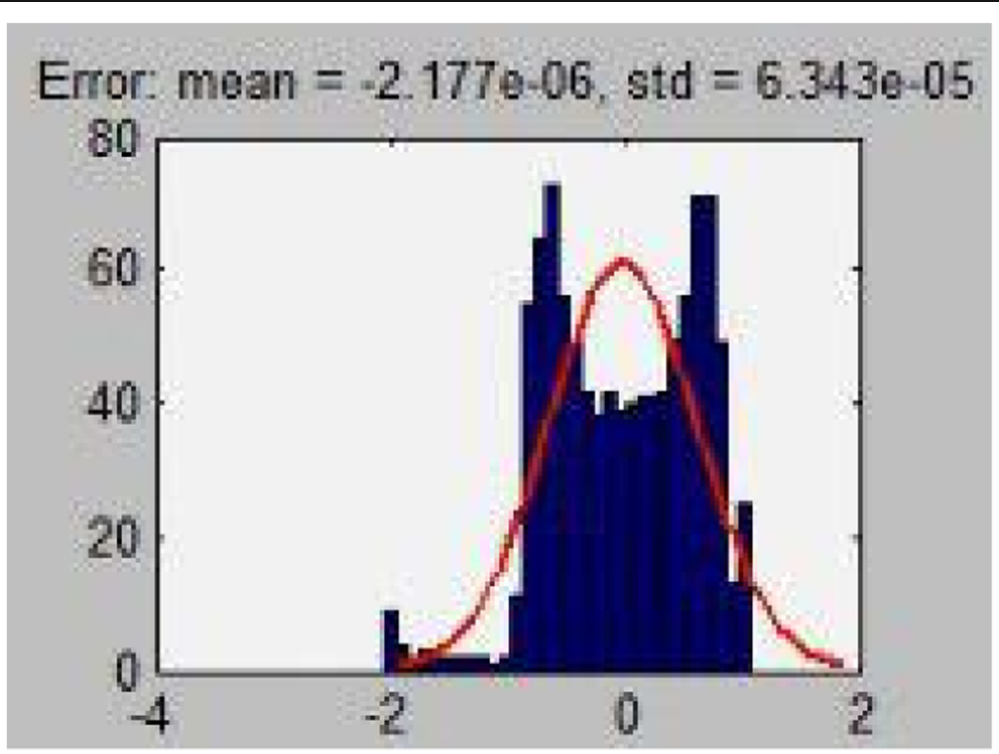

Fig. 15 The FCM MSE. There are some clustering methods like K-Means, FCM, and subtractive. To find which one is the best for our research, we have clustered the customers using K-Means, FCM, and subtractive clustering methods and compared the methods' MSEs. The MSEs (Mean-Square Error) of the FCM is shown in Fig. 15. The error mean is $=-2.177 \mathrm{e}-0.6$ and $\mathrm{std}$. $=6.343 \mathrm{e}-0.5$. The Mean squared error (MSE) measures the average of the squares of the errors

\section{Making a fuzzy inference system for the proposed model}

The fuzzy variables used to create the FIS rule base in this research were defined based on trapezoidal fuzzy numbers. As an example, consider these two factors: the number of loan repayments past due and debt-to-income ratio, as shown in Table 3. To limit the description of the value of factors, we used SD as little, MD as medium and LD as

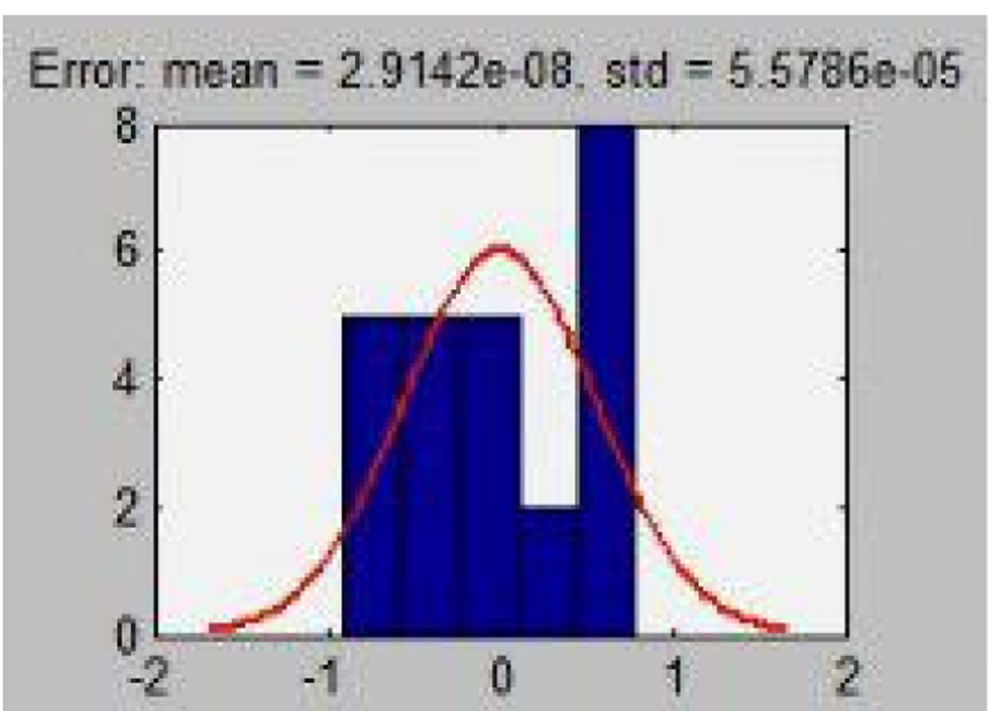

Fig. 16 The subtractive clustering MSE. There are some clustering methods like K-Means, FCM, and subtractive. To find which one is the best for our research, we have clustered the customers using K-Means, FCM, and subtractive clustering methods and compared the methods' MSEs. The MSEs (Mean-Square Error) of the subtractive is shown in Fig. 16. The error mean is $=2.9142 \mathrm{e}-0.8$ and $\mathrm{std}$. $=5.5786 \mathrm{e}-0.5$ The Mean squared error (MSE) measures the average of the squares of the errors 
Table 3 Fuzzy evaluation for debt to Income ratio and Number of Loan Repayments Past Due. This table combines two input variables. It describes that if the number of loan repayments past due is low (SD) and the amount of debt to income is low (SD) then the customer is recognized as low-risk. If the number of loan repayments past due is at around the middle (MD) and the amount of debt to income is high (LD) then the customer is recognized as the high-risk (HR). To limit the describing of the value of factors, we have used SD as little, MD as medium and LD as high

\begin{tabular}{llll}
\hline Number of loan repayment past due & SD & MD & \\
Debt to income ratio & & & \\
\hline$S D$ & $L R$ & $L R$ & $M R$ \\
$M D$ & $M R$ & $M R$ & $M R$ \\
LD & $M R$ & $H R$ & $H R$ \\
\hline
\end{tabular}

high (SD, MD, and LD are not acronyms. They are just terms that we assumed. SD stands for little, MD stands for medium, and LD stands for high).

When debt to income is greater than one it is $\mathrm{SD}$. When debt to income is equal to one it is $\mathrm{MD}$, and when debt to income is less than one it is LD. If loan repayments past due is less than two it is SD. If loan repayments past due is between two and six it is MD, and if loan repayments past due is greater than six it is LD.

Table 3 combines two input variables. It indicates that, if the number of loan repayments past due is low (SD) and the ratio of debt-to-income is low (SD), then the customer is recognized as low-risk. If the number of loan repayments past due is around the middle (MD) and the ratio of debt-to-income is high (LD) then the customer is recognized as high-risk (HR). The following are some of the rules applied according to specialist knowledge:

1. If (debt-to-income ratio is SD) and (number of loan repayments past due is SD), then (customer evaluation is MR) (1).

2. If (debt-to-income ratio is $\mathrm{SD}$ ) and (number of loan repayments past due is MD), then (customer evaluation is HR) (2).

For each factor, the membership function was defined; it is shown in Fig. 17.

The aggregation function was defined to map the input to the output, as shown in Fig. 18. Among defuzzifying methods such as "large of maximum" (LOM), "small of maximum" (SOM), and "centroid of area" (COA), COA was applied because it had the least error and the best results.

The FIS toolbox in MATLAB R2015b was used for the calculations.

\section{Statistical analysis and model estimation}

As mentioned above, the statistical population of this research includes defaulters, i.e., customers who paid their installments late: from 2 to 6 months, from 6 to 18 months, and more than 18 months late. We collected data randomly by meeting with credit experts from bank branches, examining existing archives, and monitoring the collection of claims. Thus, we used 9000 records of customers who received credit from banks from 2008 to 2016. From this collection of data, we used 7920 records to design and train the ANFIS and 1080 records to test the efficiency and predictive power of the model. Sample customers consisted of 6658 medium-risk customers (i.e., who had repaid their installments between 2 and 6 months late) and 1262 high-risk customers (who had not paid their installments for more than 6 months). Considering the number 


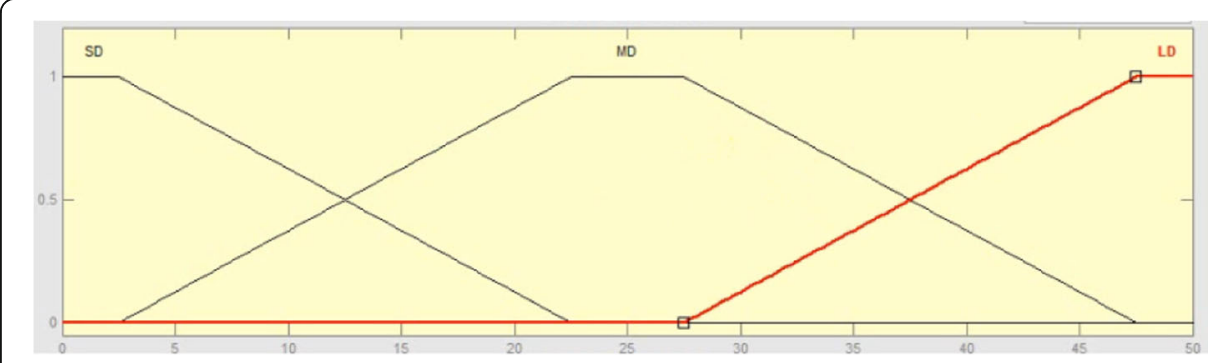

Fig. 17 Membership function of Number of Loan Repayments Past Due. The factors applied in this model as predictors are different from the previous researches. We defined these factors and a group of 10 top risk managers in several meetings approved them. Some of the factors do not change through the time, we called them certain factors. Others do, and we called them uncertain factors. We have applied fuzzy theory to uncertain factors. The fuzzy variables used to create the FIS rule base of this research were defined based on trapezoidal fuzzy numbers

of variables in the customer profile, in order to improve the accuracy of the model, it was necessary to select the most important variables to include in the model. SPSS software (IBM SPSS Statistics) (SPSS Statistics, 2009) was used to calculate correlation coefficients. Variables with the largest correlation coefficients with respect to the dependent variable (i.e. the amount of delay in installment payment) were determined. Figure 19 displays the design of the model.

In this section of the study, customer information was processed in MATLAB R2015b before entering the model. Next, 9000 records were entered into the model. Given that the range of values each variable can take was different, we normalized all data by converting them into numbers between zero and one. After this stage, the training and testing data are separately entered into the software, which then began to fit the model. Figure 20 shows the fuzzy inference system obtained in the process of training the network in MATLAB R2015b.

The parameters of the model, including the target error rate, number of repetitions, and number of fuzzy sets of each of the variables, were considered to be 0,80 , and 3 ,

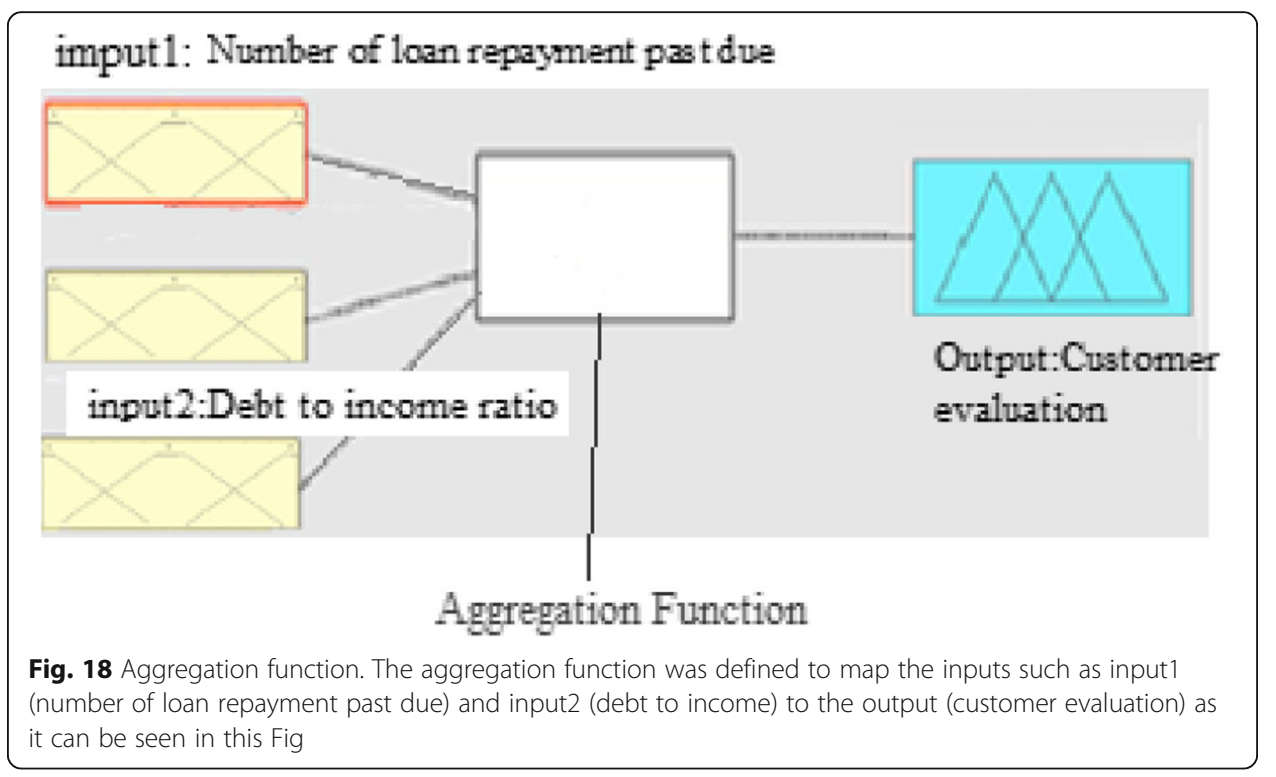




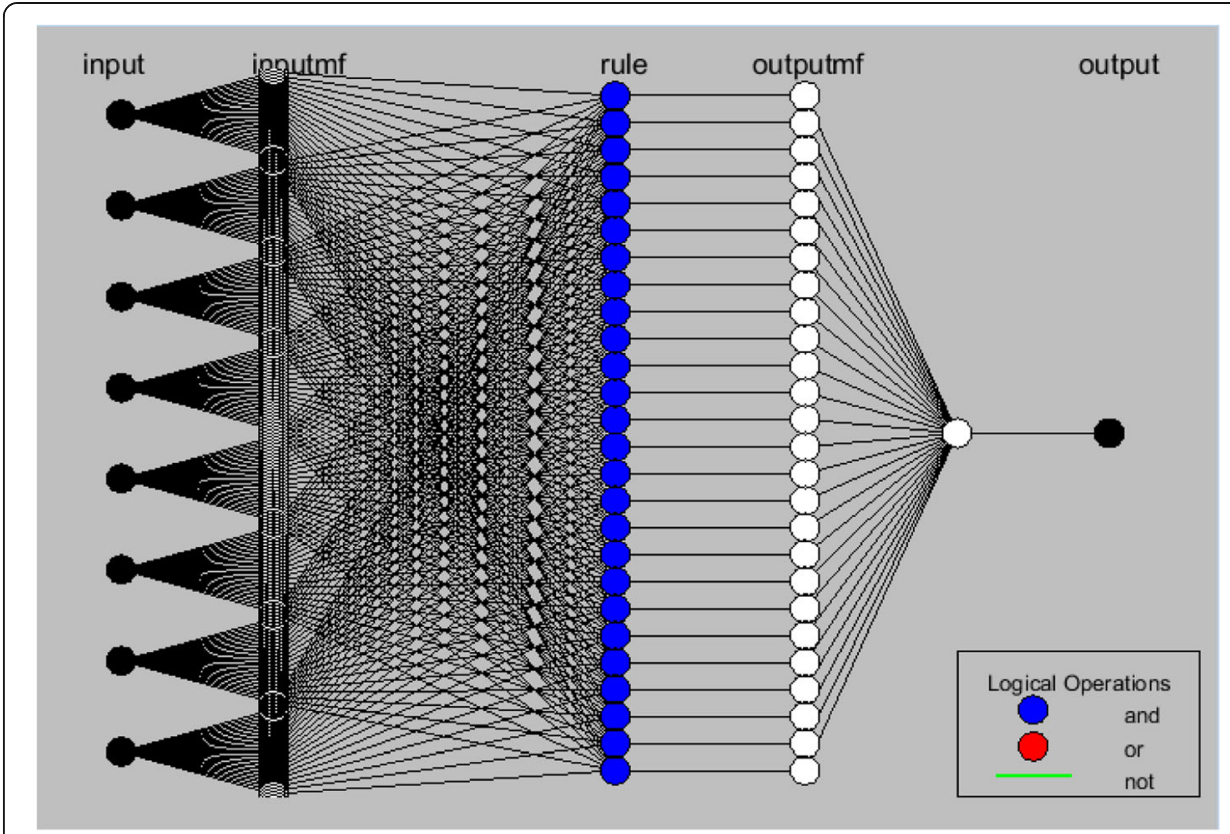

Fig. 19 Design of the model. This Figure shows the ANFIS model. It has five layers. The input layer. The left nodes are the inputs. Actually, the inputs are more than eight but it is just a sample. The second layer from the left is the inputmfs which are the membership functions of the inputs. The third layer is the rules which ANFIS find them after training. The fourth layer is outputmfs which are the membership functions of the outputs. The fifth and last layer is the output

respectively. It should be noted that the numbers of fuzzy sets for each of variables 3 and 4 were investigated. Based on the error index, fuzzy set 3 was selected. The rate of root-mean-square error (RMSE) was determined in different repetitions. The RMSE was reduced to less than 80 repetitions, after which no significant change was observed. Therefore, 80 repetitions were selected in executing the algorithm.

The performance of the model was evaluated by two indicators:

1) degree of sensitivity: the proportion of bad customers that the model classifies into the bad customer group.

2) degree of diagnosis: the proportion of well-off customers that the model classifies into the good customer group. In order to judge customers and group them into low, medium, and high risk, the probability of default within the interval $[0,1]$ was

1. If (in1 is in1cluster1) and (in2 is in2cluster1) and (in 3 is in3cluster1) and (in 4 is in4cluster1) and (in 5 is in 5 clust 2. If (in 1 is in 1 cluster 2 ) and (in 2 is in2cluster) and (in 3 is in 3cluster2) and (in 4 is in 4 cluster 2 ) and (in 5 is in 5 clust

3. If (in 1 is in1cluster3) and (in2 is in2cluster3) and (in 3 is in 3cluster3) and (in 4 is in4cluster3) and (in 5 is in 5 clust 4. If (in 1 is in 1cluster4) and (in 2 is in2cluster4) and (in 3 is in 3cluster4) and (in4 is in4cluster4) and (in 5 is in 5 clust

5. If (in 1 is in1cluster5) and (in 2 is in2cluster5) and (in 3 is in 3cluster5) and (in4 is in4cluster5) and (in 5 is in 5 clust

6. If (in 1 is in1cluster6) and (in 2 is in2cluster6) and (in 3 is in 3cluster6) and (in4 is in4cluster6) and (in 5 is in 5 clus

7. If (in1 is in1cluster7) and (in2 is in2cluster7) and (in 3 is in3cluster7) and (in4 is in4cluster7) and (in 5 is in 5 clust

8. If (in 1 is in 1cluster8) and (in 2 is in2cluster8) and (in 3 is in 3 cluster8) and (in4 is in 4 cluster 8 ) and (in 5 is in 5 clust

9 If (in1 is in1cluster9) and (in2 is in2cluster9) and (in 3 is in 3cluster9) and (in4 is in4cluster9) and (in5 is in 5clus

Fig. 20 The fuzzy Inference system of the network. Customer information was processed in Matlab R2015b before entering the model. Next, the 9000 records were entered into the model. Given that the range of the values each variable can take is different, we normalized all data by converting them into numbers between zero and one. After this stage, the training and testing data are separately entered into the software and begin to fit the model. This Fig shows the Fuzzy Inference system that is obtained in process of training the network in Matlab R2015b 
determined. If the probability of default was greater than one, the customer was considered as high-risk; a probability of less than one meant that the customer was low risk.

The optimized threshold limits of the model were assigned using the evaluation criteria. Korsholm (2004) proposed various target functions for optimization. In this study, the optimal threshold limit is equal to a value that maximizes the degree of sensitivity and the degree of diagnosis of the model.

Figure 21 shows that the optimal threshold $(\mathrm{Y})$ of the degree of sensitivity and degree of detection is 0.37 .

Table 4 shows the predicted values of the probability for the dependent variable $\mathrm{Y}$ based on being above or below the threshold in contrast with the actual values observed in the model data. It shows that the degree of sensitivity and degree of diagnosis of ANFIS in the model data were $87.08 \%$ and $91.03 \%$, respectively.

To evaluate the performance of the model, we used its predictive power for data outside the model. 54,000 records that were not used in training were entered into the model and their probability was calculated and compared with the values of the table. The results can be seen in Table 5 . The degree of sensitivity and diagnosis of the model were $0.84 .05 \%$ and $89.23 \%$, respectively.

\section{Results and discussion}

We compared the NPL rate predicted by the static models used by the banks with our proposed dynamic model; there was a significant difference $(p$ value $=0.006)$ between the predictions of the two models (Fig. 22).

Figure 22 shows the comparison between the prediction of our proposed model and the real NPL. There was little difference between them according to the proposed

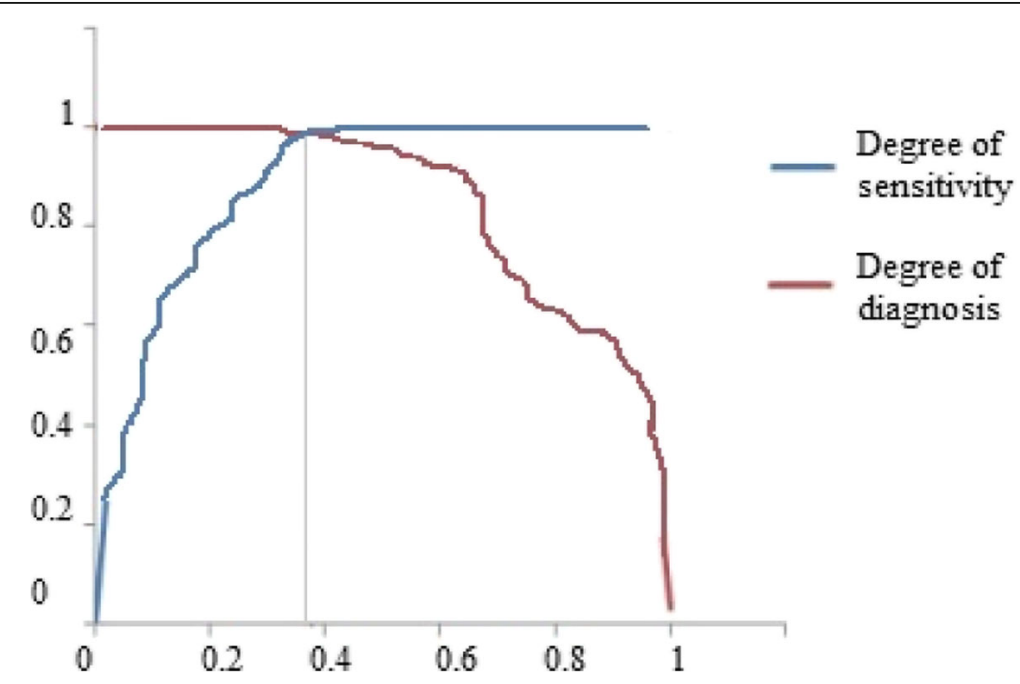

Fig. 21 The optimal threshold. The performance of the model is evaluated by two indicators: 1) Degree of sensitivity: A proportion of bad customers that the model classifies in a bad customer group. 2) The degree of diagnosis: A proportion of well-off customers that the model classifies in a good customer group. In order to judge customers and group them into low risk, medium risk, and high-risk customers, the probability of default within the interval $[0,1]$ was determined. If the probability of defaults were on more than one point, the customer was considered with a high risk and less than that means the customer is low risk. In this paper, the optimal threshold limit equals a value that maximizes the degree of sensitivity and the degree of diagnosis of the model. This Fig shows that the optimal threshold $(Y)$ of the degree of sensitivity and degree of detection is 0.37 . The blue line is the degree of sensitivity and the red line is the degree of diagnosis 
Table 4 Predictive power of the model. This table shows the predicted values of the probability for the dependent variable $Y$ based on being above or below the threshold, in contrast with the actual values observed in the model data. It shows that the degree of sensitivity and degree of diagnosis of ANFIS in the model data were $87.08 \%$ and $91.03 \%$, respectively

\begin{tabular}{llll}
\hline Real estimate observation & Medium risk customers & High risk customers & Total \\
\hline $\mathrm{P}(\mathrm{Y})<=0.37$ & 6061 & 163 & 6224 \\
$\mathrm{P}(\mathrm{Y})>0.37$ & 597 & 1099 & 1696 \\
Total & 6658 & 1262 & 7920 \\
Correct & 6061 & 1099 & 7160 \\
Correct percent & $91.03 \%$ & $87.08 \%$ & $90.04 \%$ \\
Incorrect percent & $8.96 \%$ & $12.91 \%$ & $9.5 \%$ \\
\hline
\end{tabular}

model. As an example, the average NPL was predicted as 200 billion Rials: more than the real NPL in 2012.

The ability of the new predictors to cluster customers into segments was analyzed and approved by the credit risk workgroup. Table 6 shows the dynamism of customers between segments of the bank's static model and the proposed model in terms of economic sectors. For example, it shows that, because of the sanctions, $17.1 \%$ and $5.1 \%$ of customers moved from the good segment to the bad (i.e., high-risk) segment, respectively. In the export sector, $0.2 \%$ of customers moved from the good segment to the high-risk segment. In the agriculture sector, $8.3 \%$ of customers moved from the good segment to the high-risk segment and $4.1 \%$ moved from the good segment to the medium-risk segment. In the commercial sector, $12.5 \%$ of customers moved from the good segment to the high-risk segment and $6.3 \%$ of customers moved from the good segment to the medium-risk segment. In the building sector, $9.7 \%$ of customers moved from the good segment to the high-risk segment and 3.4\% moved from the good segment to the medium-risk segment.

Morality plays an impressive role in defaults. $5 \%$ of bad debtors on high-amount loans do not suffer from any economic or other special challenge. They simply would not repay their loans before the bank forecloses.

We propose a new dynamic model for assessing the credit risk that outperforms the static models currently used, especially in the face of economic crises. Our model samples the customer database and creates a table containing data on bad customers (those with arrearage of more than 2 months) and reveals the behavioral patterns of these customers. Additionally, the model takes into account some previously neglected factors; by combining them with expert knowledge, it yields results that are closer to reality.

Table 5 Performance of the model. To evaluate the performance of the model, we use its predictive power for data outside the model. 54,000 records that were not used in training were entered into the model and their probability was calculated and compared with the values of the table. The degree of sensitivity and diagnosis of the model were $0.84 .05 \%$ and $89.23 \%$, respectively

\begin{tabular}{llll}
\hline Real estimate observation & Medium risk customers & High risk customers & Total \\
\hline$P(Y)<=0.37$ & 638 & 58 & 696 \\
$P(Y)>0.37$ & 77 & 307 & 384 \\
Total & 715 & 365 & 1080 \\
Correct & 638 & 307 & 945 \\
Correct percent & $89.23 \%$ & $84.05 \%$ & $87.5 \%$ \\
Incorrect percent & $10.76 \%$ & $84.1 \%$ & $12.5 \%$ \\
\hline
\end{tabular}




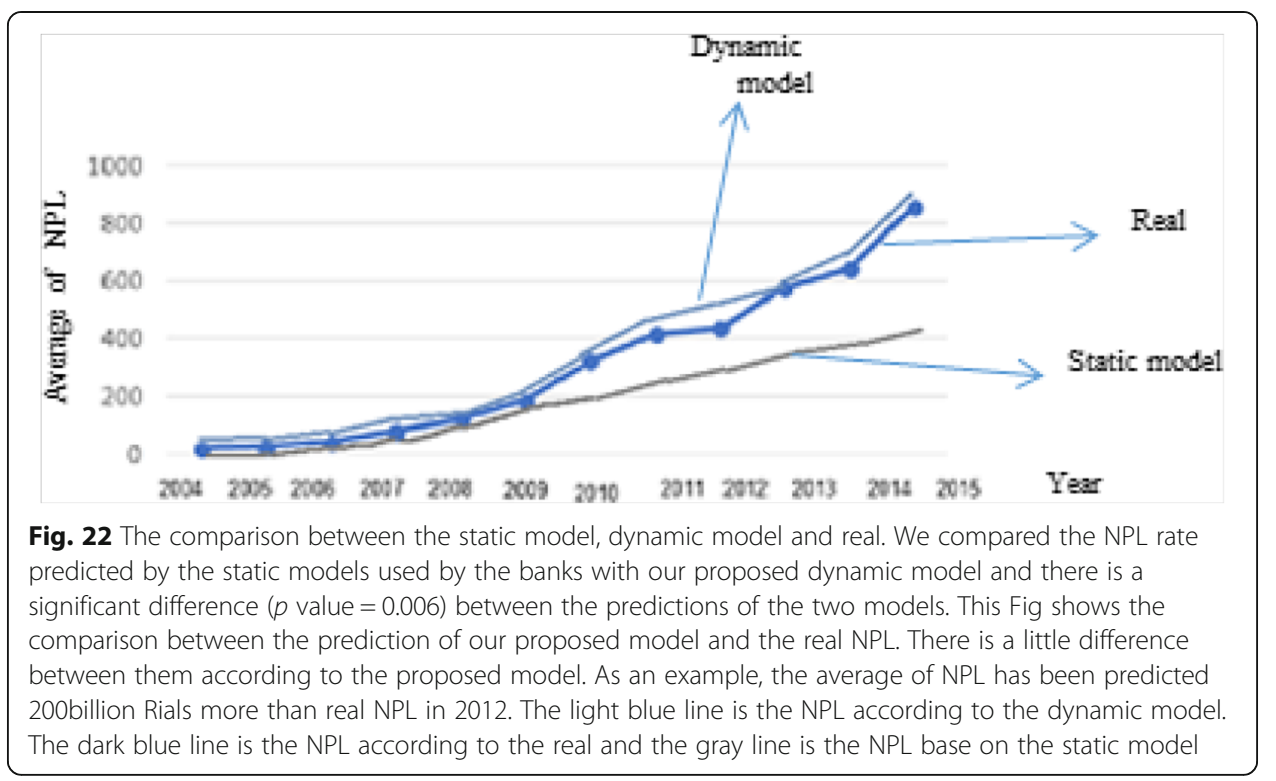

During the last decade or so, the governing regime in Iran has been under many political and economic international sanctions, which has introduced new credit risk factors. Consequently, traditional models have failed to accurately predict the behaviors of customers. This may lead to major losses on the part of banks.

Interestingly, we found that many of the defaults were among backed loans and were securitized by large collaterals. Therefore, the accuracy of the segmentations is crucial for the banks to recognize and deal with vulnerable customers. Traditional static models have proved to work reasonably well in predicting credit risks during periods of stasis, but they fail to do so in the face of economic and political fluctuations. As new factors are introduced during such a period, the model criteria need to be updated, as well. Opting for appropriate factors that work well in all circumstances is difficult (if not impossible), and a model frame that can accommodate the new factors is desirable. The credit risk workgroups can update the criteria for the model in intervals of, say, 3 months, and thus help the model to maintain its dynamism and predictions with optimum accuracy. Subsequently, banks may reserve some money for credit loss, which may help them to survive crises.

Table 6 Dynamism between segments. This table shows the dynamism of customers between segments of the bank's static model and the proposed model in terms of economic sectors. For example, it shows that because of the sanctions $17.1 \%$ and $5.1 \%$ of the customers have moved from the good segment to the bad (i.e. high-risk) segment, respectively

\begin{tabular}{|c|c|c|c|c|c|}
\hline Economic sector $\rightarrow$ & Industry & Export & Agriculture & Commerce & Building \\
\hline Percent of bad debts $\rightarrow$ & 33.7 & 1.5 & 18.6 & 26.8 & 19.4 \\
\hline $\begin{array}{c}\text { Dynamism between the good seement } \\
\text { and the current high-risk segment. }\end{array} \rightarrow$ & 17.1 & 0.2 & 8.3 & 12.5 & 9.7 \\
\hline $\begin{array}{l}\text { Dynamism between the good seement } \rightarrow \\
\text { and the current medium nisk segment. }\end{array}$ & 5.1 & - & 4.1 & 6.3 & 3.4 \\
\hline
\end{tabular}




\section{Conclusion}

In this study, we proposed a dynamic model for credit risk assessment that outperforms the models currently used. Our model has a dynamic engine that assesses the behavior of bad customers on a monthly basis and a fuzzy inference system (FIS) that includes the factors of credit risk, especially in economic crises. This model can accommodate ever-changing uncertain factors; for example, those introduced after the political and economic sanctions on the Iranian regime.

Credit scoring and prediction of loan delinquency risk have never been as important for Iranian banks as they are currently. Various models are currently used, ranging from statistical quality models such as discriminant analysis and logistic regression to comprehensive analysis of data and artificial intelligence. However, none of these approaches have taken economic and political crises into account, to our knowledge. The criteria for these models come mostly from demographic data, which normally follow a certain static pattern.

The major innovation of this research is that we sampled a customer dataset by making a table of bad customers every month and creating a dynamic model based on this data. The newly created model was then used for assessing new customers without having to repeat the whole process. The basic idea behind this survey method is that customers follow a predictable behavioral pattern in times of economic crisis. These patterns are measurable and are different from those of longer past periods of time; for example, when political and economic conditions were different. Thus, in addition to existing factors, we introduced some uncertain factors (i.e., factors that are prone to change over time) as well as some previously neglected certain factors. Unlike previous models, this characteristic of our model eliminates the impact of human judgment from the process of decision making about a loan.

This research considered uncertainty in order to develop an accurate, flexible, and dynamic model for assessing customer credit risk by combining ANFIS, fuzzy clustering, FIS, and other fuzzy theory concepts. The proposed model takes account of economic crisis in an attempt to decrease the amount of non-performing loans, to assess customer credit risk when issuing credit cards (as an economic driver in Iran), and to optimize resource allocation. Currently, twenty banks and other financial institutions are under the supervision of the Central Bank of Iran. We hope that our proposed model will replace the static models currently used in those banks. By applying this model, bankers can enter the attributes of a new customer into the dynamic model, evaluate them, and let the model make accurate decisions about them. Future research can add a set of qualitative predictors such as accountability, commitment, honesty, good reputation, and ethics to the list of risk factors used in this analysis, which may help create a model closer to reality.

The proposed model can be used for credit risk assessment in hyperinflation or bankruptcy. A group of specialists can define the effective factors affecting credit risk in these situations based on their practices or literature and then use the proposed dynamic model to assess credit risk.

Attention to a single counterparty in defining the effective factors impacting credit risk may be the subject of further research. The dynamic credit risk assessment model can be specialized for two categories of loans: one for loans like credit cards and the other for loans like mortgages. Considering loan terms and amounts may yield different results. 


\section{Abbreviations}

ANFIS: Adaptive Network-based Fuzzy Inference System; ANN: Artificial Neural Network; BN: Bayesian networks; FCM: Fuzzy C-Means; FIS: Fuzzy Inference System; FS: Feature Selection; GA: Genetic Algorithm; GAM: Generalized Additive Model; HR: High Risk; K-NN: K-Nearest Neighbors; LGD: loss given default; LR: Low Risk; MDA: Multiple Discriminant Analysis; MLP: Multilayer Perceptron Network; MR: Medium Risk; MSE: Mean Squared Error; NN: Neural Networks; NPL: Non-Performing Loan; PCA: Principal Component Analysis; RBF: Radial Base Function; RMSE: Root-MeanSquare Error; SVM: Support Vector Machine

\section{Acknowledgements}

Not applicable.

\section{Funding}

This research did not receive any specific grant from funding agencies in the public, commercial, or not-for-profit sectors.

\section{Availability of data and materials}

We have used a customer dataset of a bank which emphasized data and bank name must be kept confidential.

\section{Authors' contributions}

Both authors read and approved the final manuscript

\section{Competing interests}

The authors declare that they have no competing interests.

\section{Publisher's Note}

Springer Nature remains neutral with regard to jurisdictional claims in published maps and institutional affiliations.

\section{Author details}

${ }^{1}$ Science and Research Branch, Islamic Azad University, Tehran, Iran. ${ }^{2}$ Tarbiat Modares University, Tehran, Iran.

Received: 21 November 2017 Accepted: 18 January 2019

Published online: 20 March 2019

\section{References}

Abdipour S, Nasseri A, Akbarpour M, Parsian H, and Zamani S (2013) Integrating neural network and colonial competitive algorithm: a new approach for predicting bankruptcy in tehran security exchange. Asian Econ Fin Rev 3(11):1528-1539. Abdou H, Pointon J (2009) Credit scoring and decision making in Egyptian public sector banks. Int J Manag Finance 5(4):391-406 Akkoc S (2012) An empirical comparison of conventional techniques, neural networks and the three stage hybrid adaptive neuro fuzzy inference system (ANFIS) model for credit scoring analysis: the case of Turkish credit card data. Eur J Operat Res 222(1):168-178

Amid A. (n.d.) Fuzzy logic, in press

Baixauli J, Alvarez S, Módica A (2012) Combining structural models and accounting based models for measuring credit risk in real estate companies. I J Manag Finance 8(1):73-95.

Bekhet HA, and Eletter SFK, (2014) Credit risk assessment model for Jordanian commercial banks: Neural scoring approach. Review of Development Finance. 4(1):20-28.

Baradaran V, and Keshavarz M (2015) An integrated approach of system dynamics simulation and fuzzy inference system for retailers' credit scoring. Economic research - Ekonomska istraživanja. 28(1):959-980. https://doi.org/10.1016/j.ejor.2012.04.009.

Bensic M, Sarlija N, Zekic-Susac M (2005) Modeling small-business credit scoring by using logistic regression, neural networks and decision trees. Intelligent Syst Account Fin Manage 13(3):133-150.

Berthold MR, Hand DJ (2003) Intelligent data analysis: an introduction. 2nd. Springer-Verlag Berlin Heidelberg, New York.

Blanco A, Pino-Mejías R, Lara J, Rayo S (2013) Credit scoring models for the microfinance industry using neural networks: Evidence from Peru. Expert Systems with Applications. 40(1);356-364.

Chen W, Xiang G, Liu Y, Wang K (2012) Credit risk evaluation by hybrid data mining technique. Syst Eng Procedia 3:194-200.

Chi Q, Li W (2017) Economic policy uncertainty, credit risks and banks'lending decisions: Evidence from Chinese commercial banks. China J Account Res 10(1):33-50

Cisko Š, Klieštik T. Finančný manažment podniku II. Žilina (2013) EDIS Publishers, University of Žilina.

Danenas P, and Garsva G (2015) Selection of Support Vector Machines based classifiers for credit risk domain. Expert Syst. Appl. 42(6):3194-3204.

Dikjkman JG, Vanhaeringen haerongen H, Delanges SJ (1983) Fuzzy Numbers. J Math Anal Appl 92(2):301-341.

Emel A, Oral M, Reisman A, Yolalan R (2003) A credit scoring approach for the commercial banking sector. Socio Econ Plan Sci 37(2):103-123.

Ghodselahi A (2011) A Hybrid Support Vector Machine Ensemble Model for Credit Scoring. International Journal of Computer Applications 17:0975-8887.

Girjia N, Sirvatsa SK (2006) A research study: using data mining in knowledge base business strategies. Inf Technol J 7(2):590-600.

Heiat A (2012) Comparing performance of data mining models for computer credit scoring. J Int Fin Econ 12(1):78-83.

Hsieh NC, Hung LP (2010) A data driven ensemble classifier for credit scoring analysis. Expert Syst Appl 37(1):534-545.

Jang J-SR (1993) ANFIS: adaptive-network-based fuzzy inference systems. IEEE Trans Syst Man Cybernetics 23(3):665-685.

Kaufman L, Rousseeuw PJ (2009) Finding groups in data: an introduction to cluster analysis. Wiley, New York.

Khandani A, Kim A, Lo A (2010) Consumer credit-risk model via machine learning algorithms. J Bank Finance 34(11):2767-2787. 
Korsholm, L (2004), Analysis of diagnostic studies, sensitivity and specificity positive predicted values ROC curves tests based on logistic regression. Department of statistics and demography, University of Southern Denmark.

Kruppa J, Schwarz AG, Ziegler A (2013) Customer credit risk: individual probability estimates using machine learning. Expert Syst Appl 40(13):5125-5131.

MATLAB And statistics toolbox release R2015b, the math works, Inc., Natick, Massachusetts, United States (n.d.).

Nanni L, Lumini A (2009) An experimental comparison of ensemble of classifiers for bankruptcy prediction and credit scoring. Expert Syst Appl 36(2):3028-3033.

Mandala I.G.N.N., Nawangpalupi C. A., and Praktikto F. R (2012) Assessing Credit Risk: An Application of Data Mining in a Rural Bank. Procedia Economics and Finance. 4:406-412.

Nauk D, Klawonn F, Kruse R (1997) Foundations of neuro-fuzzy systems. Willey, New York.

Koutanaei F.N., Sajedi H, and Khanbabaei M (2015) A hybrid data mining model of feature selection algorithms and ensemble learning classifiers for credit scoring. J Retail Consum Serv 27:11-23.

Oreski S, and Oreski G, (2014) Genetic algorithm-based heuristic for feature selection in credit risk assessment. Expert Syst Appl 41(4):2052-2064

Paleologo G, Elisseeff A, and Antonini G (2010) Subagging for credit scoring models. Eur J Oper Res 201(2);490-499

Ping Y, Yongheng L (2011) Neighborhood rough set and SVM based hybrid credit scoring classifier. Expert Syst Appl 38(9): $11300-11304$

Polat K, Günes S (2006) an expert system approach based on principal component analysis and adaptive neuro-fuzzy inference system to diagnosis of diabetes disease. Digital Signal Process 17(4):702-710.

Procter P (1978). Longman dictionary of contemporary English. Harlow [England], Longman.

Rao HS, Mukherjee A (1996) Artificial neural networks for predicting the macro mechanical behavior of ceramic-matrix composites. Comput Mater Sci 5(4):307-322.

Rosenblatt F (1958) The perceptron: a probabilistic model for information storage and organization in the brain. Psychol Rev 65(6):38-65.

Saitta S, Kripakaran P, Raphael B, Smith IF (2008) Improving system identification using clustering. J Comput Civ Eng 22(5):292-302.

Shahari F, Zakaria R, Rahman S (2015) Investigation of the expected loss of sharia credit instruments in global Islamic banks. Int J Manag Finance 11(4):503-512.

Sousa M R, Gama J and Brandão E. A new dynamic modeling framework for credit risk assessment. Expert Syst Appl 45:341-351.

Sugeno, M (1985). Industrial applications of fuzzy control. Elsevier Science Ltd; First Edition.

Sumathi S, Sivanandam SN (2006) Introduction to data mining and its applications. Springer-Verlag, Berlin.

SPSS Statistics is a software package used for logical batched and non-batched statistical analysis. Long produced by SPSS Inc., it was acquired by IBM in 2009. The current versions are officially named IBM SPSS Statistics.

Tsai CFT (2008) Using neural network ensembles for bankruptcy prediction and credit scoring. Expert Syst Appl 34(4):2639-2649.

Twala B (2010) Multiple classifier application to credit risk assessment. Expert Syst Appl 37(4):3326-3336.

Wang G, Hao J, Ma J, Jiang H (2011) A comparative assessment of ensemble learning for credit scoring. Expert Syst Appl 38(1):223-230

Wang G, Ma J, Yang S (2014) An improved boosting based on feature sekection for corporate bankruptcy prediction. Expert Syst Appl 41(5):2353-2361.

Wang Y, Chen Y (2014) A comparison of madman and Sugeno fuzzy inference systems for traffic flow prediction. J Comput 9(1):12-21.

West D (2000) Neural network credit scoring models. Comp Operat Res 2000:1131-1152.

Witkowska D (2006) Discrete Choice Model Application to the Credit Risk Evaluation. Int. Adv. Econ. Res. 12(1)33-42.

Yeh IC and Lien CH. The comparisons of data mining techniques for the predictive accuracy of probability of default of credit card clients. Expert Syst Appl 36(2):2473-2480.

Yurdakul F (2014) Macroeconomic modelling of credit risk for banks. Procedia Soc Behavioral 109:784-793.

Zamani S (2011) Evaluating of predicting power of ANN in order to predict customer's credit risk. Thesis.

Zanin M et al (2016) Combining complex networks and data mining: why and how. Phys Rep 635:1-44.

Zhiwang Zhang, Guangxia Gao,and Yong Shi (2014) Credit risk evaluation using multi-criteria optimization classifier with kernel, fuzzification and penalty factors. Eur. J. Oper. Res. 237(1):335-348.

\section{Submit your manuscript to a SpringerOpen ${ }^{\circ}$ journal and benefit from:}

- Convenient online submission

- Rigorous peer review

- Open access: articles freely available online

High visibility within the field

- Retaining the copyright to your article

Submit your next manuscript at $\boldsymbol{\nabla}$ springeropen.com 